Letter Report

Environmental Monitoring Systems Initiative

\title{
Twelve Months of Air Quality Monitoring at Ash Meadows National Wildlife Refuge, Southwestern Rural Nevada, U.S.A.
}

\author{
prepared by \\ Johann P. Engelbrecht, Ilias Kavouras, David S. Shafer, Dave Campbell, \\ Scott Campbell, Greg McCurdy, Steven D. Kohl, George Nikolich, \\ Larry Sheetz and Alan W. Gertler \\ Desert Research Institute \\ Nevada System of Higher Education \\ submitted to \\ Nevada Site Office \\ National Nuclear Security Administration \\ U.S. Department of Energy \\ Las Vegas, Nevada
}

August 2011

The work upon which this report is based was supported by the U.S. Department of Energy under Contract \#DE-AC52-06NA26383. 
Reference herein to any specific commercial product, process, or service by trade name, trademark, manufacturer, or otherwise, does not necessarily constitute or imply its endorsement, recommendation, or favoring by the United States Government or any agency thereof or its contractors or subcontractors. The views and opinions of authors expressed herein do not necessarily state or reflect those of the United States Government or any agency thereof.

This report has been reproduced directly from the best available copy.

Available for sale to the public, in paper, from:

U.S. Department of Commerce

National Technical Information Service

5285 Port Royal Rd.

Springfield, VA 22161

phone: 800.553.6847

fax: 703.605.6000

email: order@ntis.fedworld.gov

online ordering: http://www.ntis.gov/ordering.htm

Available electronically at http://www.doe.gov/bridge

Available for a processing fee to the U.S. Department of Energy and its contractors, in paper, from:

U.S. Department of Energy

Office of Scientific and Technical Information

P.O. Box 62

Oak Ridge, TN 37831-0062

phone: 423.576 .8401

fax: 423.576 .5728

email: reports@adonis.osti.gov 


\section{EXECUTIVE SUMMARY}

The one year of air quality monitoring data collected at the Ash Meadows National Wildlife Refuge (NWR) was the final part of the air quality "Scoping Studies" for the Environmental Monitoring Systems Initiative (EMSI) in southern and central Nevada. The objective of monitoring at Ash Meadows was to examine aerosol and meteorological data, seasonal trends in aerosol and meteorological parameters as well as to examine evidence for long distance transport of some constituents.

The 9,307 hectare refuge supports more than 50 springs and 24 endemic species, including the only population of the federally listed endangered Devil's Hole pupfish (Cyprinodon diabolis) (U.S. Fish and Wildlife Service, 1990). Ash Meadows NWR is located in a Class II air quality area, and the aerosol measurements collected with this study are compared to those of Interagency Monitoring of Protected Visual Environments (IMPROVE) sites. Measurements taken at Ash Meadows NWR over a period of 12 months provide new baseline air quality and meteorological information for rural southwestern Nevada, specifically Nye County and the Amargosa Valley.

All mass measurements fall well below the US EPA National Ambient Air Quality Standards (NAAQS) for $\mathrm{PM}_{10}\left(150 \mu \mathrm{g} / \mathrm{m}^{3}\right.$ for $24 \mathrm{hr}$ averaging interval) and $\mathrm{PM}_{2.5}\left(35 \mu \mathrm{g} / \mathrm{m}^{3}\right.$ for $24 \mathrm{hr}$ averaging interval, $15 \mu \mathrm{g} / \mathrm{m}^{3}$ for annual averaging interval). The averaged levels measured on Teflon ${ }^{\circledR}$ filters at Ash Meadows NWR for both $\mathrm{PM}_{10}\left(12 \mu \mathrm{g} / \mathrm{m}^{3}\right)$ and $\mathrm{PM}_{2.5}\left(5 \mu \mathrm{g} / \mathrm{m}^{3}\right)$, are similar to those previously measured at four rural IMPROVE sites.

The mineral dust (soil) components, including the silicate oxides, minor oxides and gypsum, together make up the largest percentage of the aerosol, being on average $58 \%$ of $\mathrm{PM}_{10}$ by mass and $37 \%$ of $\mathrm{PM}_{2.5}$ by mass, the difference being ascribed to the coarser nature of the soil forming minerals.

The highest concentrations of mineral dust components were measured during drier fall months of October and November, 2009. Although halite $(\mathrm{NaCl})$ was found at all sites, the percentages were always very low, except for the filter samples collected on 10/04/2009. The anomalously high values of $8 \%$ halite for $\mathrm{PM}_{10}$ and $10 \%$ for $\mathrm{PM}_{2.5}$ points to an evaporite (salt) source such as an alkaline playa dust. Playas are common in the region around Ash Meadows NWR.

Although secondary ammonium sulfate concentrations vary with season, they are similar for $\mathrm{PM}_{10}$ and $\mathrm{PM}_{2.5}$, implying that this species occurs in the finer fraction. Average values for $\mathrm{PM}_{10}$ were $0.5 \mu \mathrm{g} / \mathrm{m}^{3}$, and $0.7 \mu \mathrm{g} / \mathrm{m}^{3}$ for $\mathrm{PM}_{2.5}$, with the highest concentrations in spring, 2009 and the lowest in the following late fall and winter. Secondary ammonium sulfate is from $\mathrm{SO}_{2}$ emitted by distant power plants and other combustion sources, and transported into the Amargosa Valley. 
Secondary ammonium nitrate concentrations fluctuate by month, with both the lowest $\left(0.08 \mu \mathrm{g} / \mathrm{m}^{3}\right.$ for $\mathrm{PM}_{10}$ and $0.03 \mu \mathrm{g} / \mathrm{m}^{3}$ for $\left.\mathrm{PM}_{2.5}\right)$ and highest $\left(1.11 \mu \mathrm{g} / \mathrm{m}^{3}\right.$ for $\mathrm{PM}_{10}$ and 0.77 $\mu \mathrm{g} / \mathrm{m}^{3}$ for $\mathrm{PM}_{2.5}$ ) measured values occurring in fall of 2009. Secondary nitrates form from nitrogen oxides emitted from vehicle emissions, possibly transported to Amargosa Valley from the Las Vegas - Los Angeles corridor.

Elemental carbon (EC) is generally very low (on average $<0.2 \mu \mathrm{g} / \mathrm{m}^{3}$ ) except for the sample taken on 8/29/2009 when high values for EC of $1.20 \mu \mathrm{g} / \mathrm{m}^{3}$ for $\mathrm{PM}_{10}$ and $1.04 \mu \mathrm{g} / \mathrm{m}^{3}$ for $\mathrm{PM}_{2.5}$ were measured. Similarly, OC remains low (on average $<1.8 \mu \mathrm{g} / \mathrm{m}^{3}$ ) except for the sample taken on $8 / 29 / 2009$ when high values for OC of $8.5 \mu \mathrm{g} / \mathrm{m}^{3}$ for $\mathrm{PM}_{10}$ and $7.5 \mu \mathrm{g} / \mathrm{m}^{3}$ for $\mathrm{PM}_{2.5}$ were measured. These high carbon measurements in the last week of August, 2009 are ascribed to woodsmoke, transported from wildfires in California. 


\section{CONTENT}

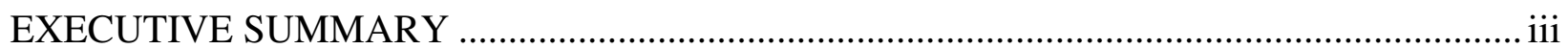

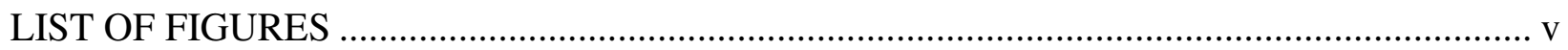

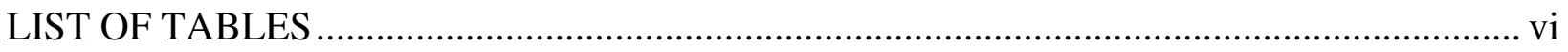

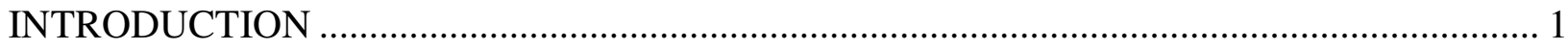

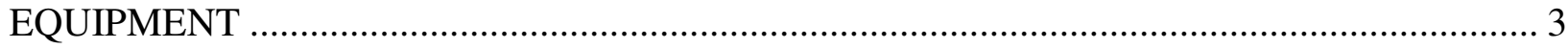

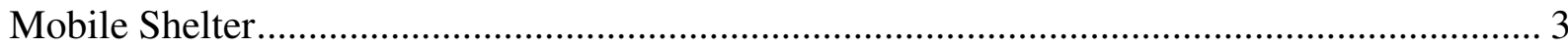

Aerosol Monitors ..................................................................................................... 4

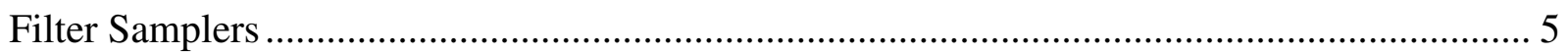

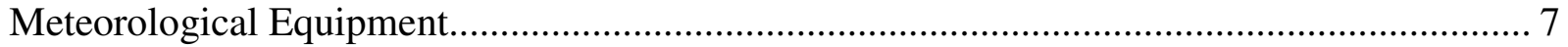

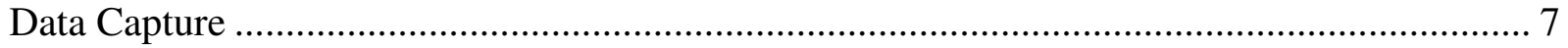

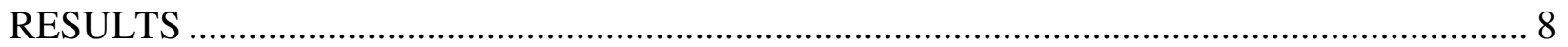

Continuous PM \& Meteorological Monitoring.......................................................... 8

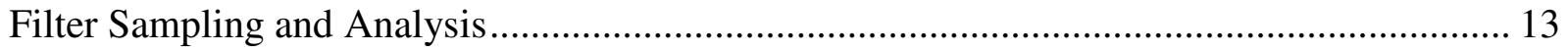

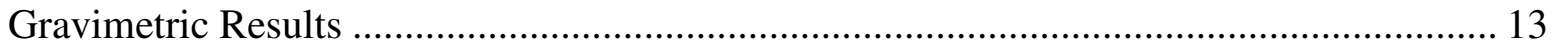

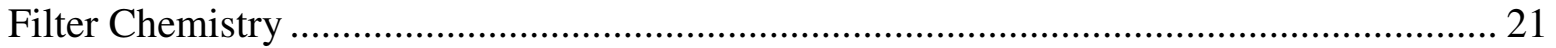

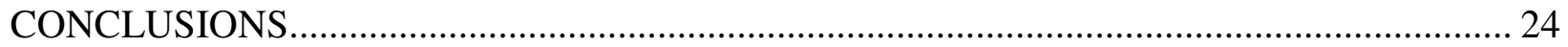

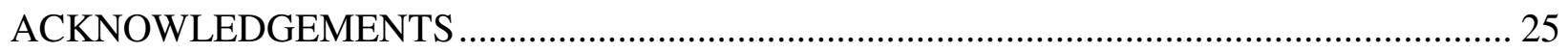

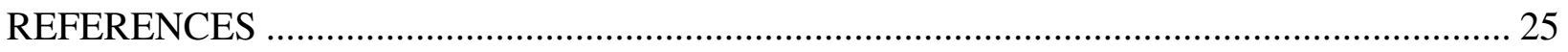

\section{LIST OF FIGURES}

1. Location of the Ash Meadows National Wildlife Refuge (NWR) in southwestern Nevada, in relation to Death Valley National Park as well as surrounding urban and rural areas.

2. Locality map showing the Farmhouse and Administrative sampling and monitoring sites at the Ash Meadows National Wildlife Refuge in Amargosa Valley, southwestern Nevada.

3. Instrumented mobile shelter at Ash Meadows NWR, showing the meteorological tower with instruments, aerosol sampling inlets, and solar panels. .............................................. 4

4. Two rack mounted TEOM ${ }^{\circledR}$ continuous particulate matter (PM) monitors in the mobile shelter, $\mathrm{PM}_{2.5}$ to the left, $\mathrm{PM}_{10}$ to the right.

5. Two BGI PQ200 ${ }^{\circledR}\left(\mathrm{PM}_{2.5}\right)$ (foreground), and one BGI PQ100 ${ }^{\circledR}\left(\mathrm{PM}_{10}\right)$ (green top box in far background) filter samplers mounted on a counter top in the mobile shelter. .................. 6

6. Wind rose shows the predominant wind from the south-southeast for summer $2009 \ldots \ldots \ldots \ldots . . .9$

7. Wind rose shows the predominant wind from the southeast but also with weaker northern to northwesterly and easterly winds in fall 2009. 
8. Wind rose shows similar winds from the southeast and northwest, with periods from the east, during winter 2009/10......

9. Wind rose shows the predominant wind from the southeast and to a lesser degree from the northwest, for spring 2010.

10. $\mathrm{PM}_{10}$ (gray) and $\mathrm{PM}_{2.5}$ (black) gravimetric data as measured on Teflon ${ }^{\circledR}$ filters, collected on a 1-in-12 day schedule, for 14 individual sampling days.

11. Hysplit backward trajectory model showing airflow from California towards Ash Meadows NWR for the August 27-29 period, coinciding to reported large wildfires in Los Angeles County during that period.

12. Chemical measurements combined as silicate oxides, minor metal oxides, ammonium sulfate, ammonium nitrate, gypsum, halite, sodium sulfate, potassium sulfate, elemental carbon (EC) and organic carbon (OC), for $\mathrm{PM}_{10}$ and associated $\mathrm{PM}_{2.5}$ size fractions. Sample labels include the sample numbers, sampling dates, and particulate size cut.

\section{LIST OF TABLES}

1. Localities of two sites at Ash Meadows NWR sampled over the course of approximately one year.

2. Equipment installed on or inside the mobile air quality shelter......

3. Average $\mathrm{PM}_{10}$ and $\mathrm{PM}_{2.5}$ gravimetric results measured at Ash Meadows NWR, by TEOM $^{\circledR}$ continuous monitors, as well as US EPA National Ambient Air Quality Standards (NAAQS) for particulate matter.

4. Statistics of continuous particulate mass (PM) and meteorological monitoring, as well as Teflon filter measurements, for summer 2009.

5. Statistics of continuous particulate mass (PM) and meteorological monitoring, as well as Teflon filter measurements, for fall 2009.

6. Statistics of continuous particulate mass (PM) and meteorological monitoring, as well as Teflon filter measurements, for winter 2009/10.

7. Statistics of continuous particulate mass (PM) and meteorological monitoring, as well as Teflon filter measurements, for spring 2010.

8. Chemical and normative mineral compositions for spring and summer 2009 samples. ........ 14

9. Chemical and normative mineral compositions for summer and fall 2009 samples............ 15

10. Chemical and normative mineral compositions for fall 2009 samples.............................. 16

11. Chemical and normative mineral compositions for fall 2009, winter 2009/10 and spring 2010 samples.

12. Chemical and normative mineral compositions for spring 2010 samples

13. Comparison of seasonal average gravimetric results from this study (Ash Meadows NWR, 05/25/09 to 05/25/10) with those from the preceding study at eight sites in rural southwestern Nevada, urban southwestern U.S.A. (CSN) and rural southwestern U.S.A. (IMPROVE) sites. 


\section{INTRODUCTION}

The one year of air quality monitoring data collected at the Ash Meadows National Wildlife Refuge (NWR) was the final part of the air quality "Scoping Studies" for the Environmental Monitoring Systems Initiative in southern and central Nevada. Results of six to nine week monitoring campaigns were previously published from eight sites in Nye and Lincoln counties, including an earlier collection phase at Ash Meadows NWR [Engelbrecht et al. 2007a]; Beatty [Engelbrecht et al. 2007b], Rachel [Engelbrecht et al. 2007c], Sarcobatus Flat [Engelbrecht et al. 2007d], Caliente [Engelbrecht et al. 2008a], Crater Flat [Engelbrecht et al. 2008b] Pahranagat NWR [Engelbrecht et al. 2008c] and Tonopah [Engelbrecht et al. 2008d]. In addition, unpublished data were collected from Lower Forty Mile Wash on the Nevada National Security Site. The objectives of monitoring at Ash Meadows were to examine aerosol and meteorological data, seasonal trends, as well as to examine evidence for long-distance transport of some constituents. The instrumented trailer was deployed at Ash Meadows from May 20, 2009 until June 4, 2010.

Ash Meadows NWR is in the Amargosa Valley in Nye County in southern Nevada (36 $25^{\circ} \mathrm{N}, 116^{\circ} 20^{\prime} \mathrm{W}, 750$ meter elevation), 145 kilometers $(\mathrm{km})$ northwest of Las Vegas and approximately $65 \mathrm{~km}$ east of Death Valley National Park (Trammell et al., 2008) (Figure 1).

Located in the northern Mojave Desert, Ash Meadows NWR is a wetland complex created by a series of faults that force groundwater from the Death Valley Regional Flow System to the surface (Belcher, 2004). Based on a 10 year record from the a meteorological monitoring station in Amargosa Valley, the area average annual precipitation is $95 \mathrm{~mm}$ (3.74 inches), with the highest monthly averages occurring in January and February (Shafer and Hartwell, 2011). The warmest month is July with an average daily maximum temperature of $40.2{ }^{\circ} \mathrm{C}\left(104.3{ }^{\circ} \mathrm{F}\right)$. December, on average, is the coolest month, with average lows being just above freezing $\left(0.2{ }^{\circ} \mathrm{C}\right.$ or $32.3^{\circ} \mathrm{F}$ ). The 9,307 hectare refuge supports more than 50 springs and 24 endemic species, including the only population of the federally listed endangered Devil's Hole pupfish (Cyprinodon diabolis) (U.S. Fish and Wildlife Service, 1990). Ash Meadows NWR is designated a Class II air quality area, and particulate matter (PM) levels are compared to those at Interagency Monitoring of Protected Visual Environments (IMPROVE) (http://vista.cira.colostate.edu/IMPROVE/Overview/Overview.htm) sites in the region. 


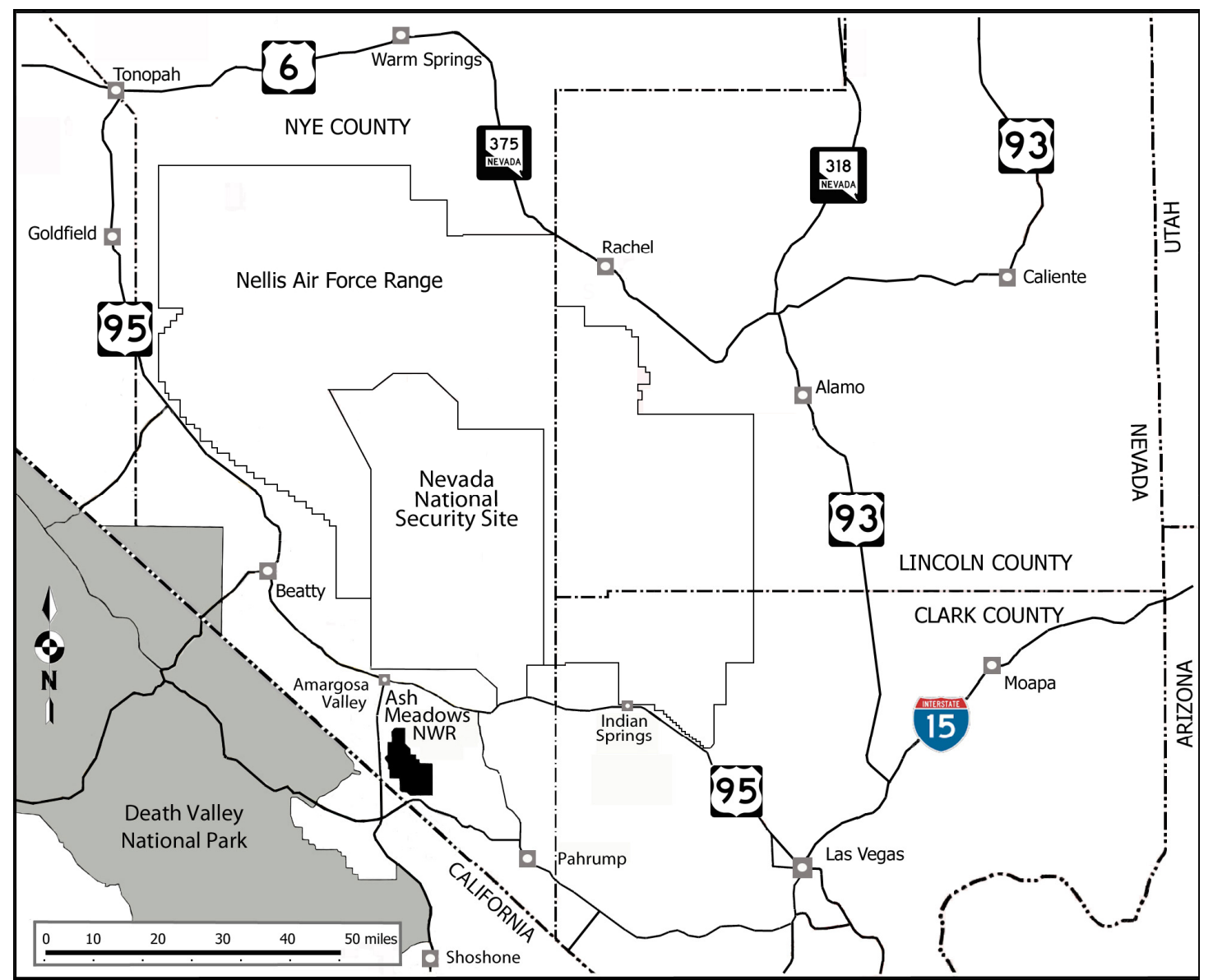

Figure 1. Location of the Ash Meadows National Wildlife Refuge (NWR) in southwestern Nevada, in relation to Death Valley National Park as well as surrounding urban and rural areas.

Monitoring was performed at two sites on the refuge, first for about eight months at an abandoned farmhouse [Engelbrecht et al. 2007a], and then for the remaining four months at the Ash Meadows NWR administrative complex (Table 1, Figure 2), $5.4 \mathrm{~km}$ northwest of the first site.

Table 1. Localities of two sites at Ash Meadows NWR sampled over the course of approximately one year

\begin{tabular}{|c|c|c|c|c|c|}
\hline Site \# & Locality & Coordinates & $\begin{array}{l}\text { Elevation } \\
\text { (m) }\end{array}$ & $\begin{array}{c}\text { Date Start } \\
(\mathbf{m} / \mathbf{d} / \mathbf{y r})\end{array}$ & $\begin{array}{c}\text { Date End } \\
(\mathbf{m} / \mathbf{d} / \mathbf{y r})\end{array}$ \\
\hline $1 \mathrm{a}$ & Farmhouse site & 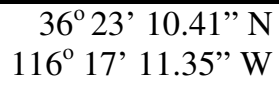 & 858 & $5 / 20 / 09$ & $1 / 26 / 10$ \\
\hline $1 b$ & Administration site & $\begin{array}{r}36^{\circ} 25^{\prime} 12.19^{\prime \prime} \mathrm{N} \\
116^{\circ} 19^{\prime} 48.50 ” \mathrm{~W}\end{array}$ & 750 & $1 / 26 / 10$ & $6 / 4 / 10$ \\
\hline
\end{tabular}




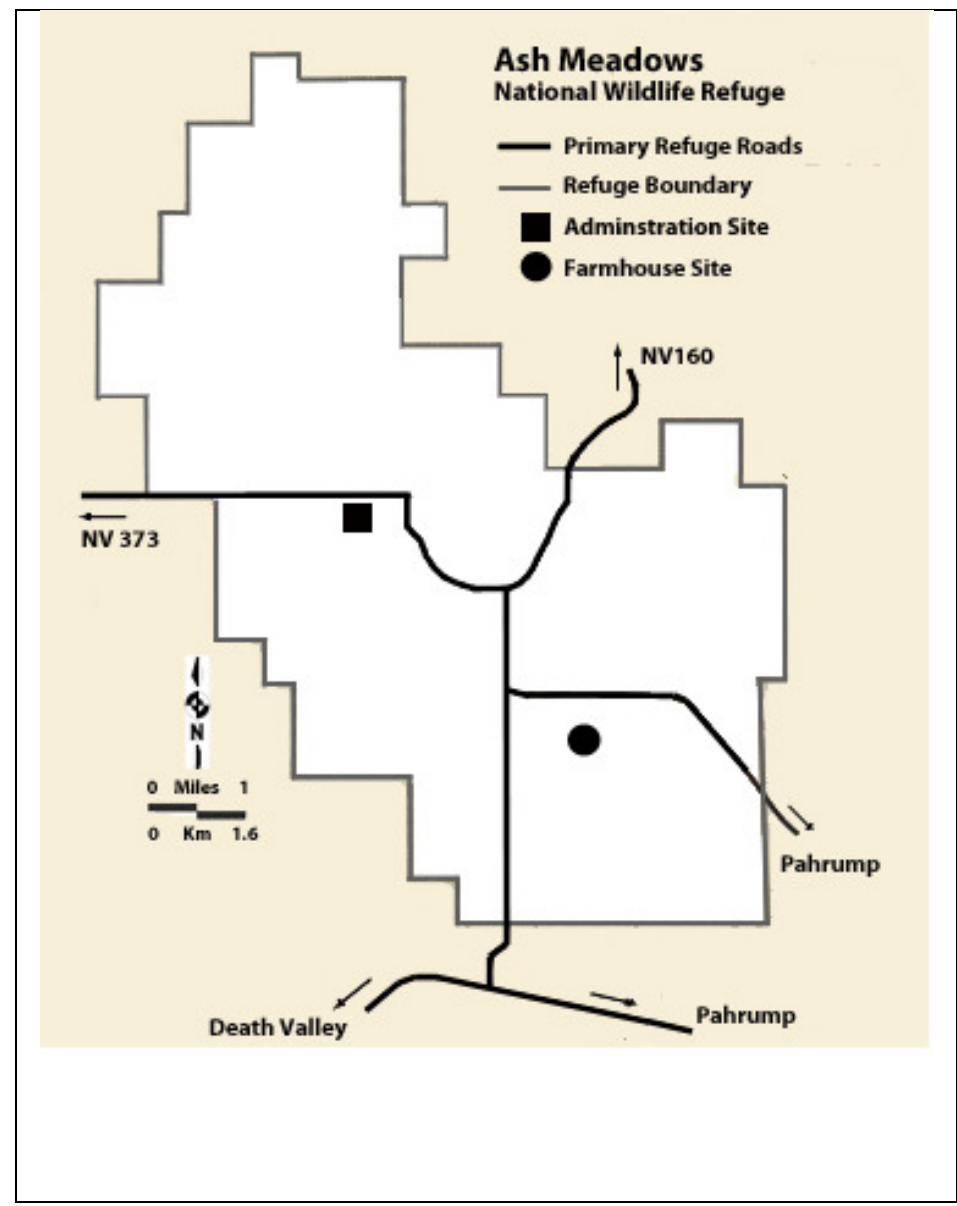

Figure 2. Locality map showing the Farmhouse $(\bullet)$ and Administrative (घ) sampling and monitoring sites at the Ash Meadows National Wildlife Refuge in Amargosa Valley, southwestern Nevada.

\section{EQUIPMENT}

\section{Mobile Shelter}

The equipment in the instrumented mobile shelter (Figure 3) was normally powered off $120 \mathrm{~V} \mathrm{AC}$, and where not available, off $12 \mathrm{~V} \mathrm{DC}$ batteries that are charged by solar panels. A retractable tower was mounted with an array of meteorological equipment, including wind, precipitation, temperature, and solar radiation monitors. Instruments housed inside the trailer included rack mounted continuous aerosol monitors and sequential filter samplers. The trailer was equipped with a data logger and a satellite communication system. Continuous data were logged and transmitted via a Geostationary Operational Environmental Satellite (GOES) to the Western Regional Climate Center (WRCC) at DRI in Reno. 


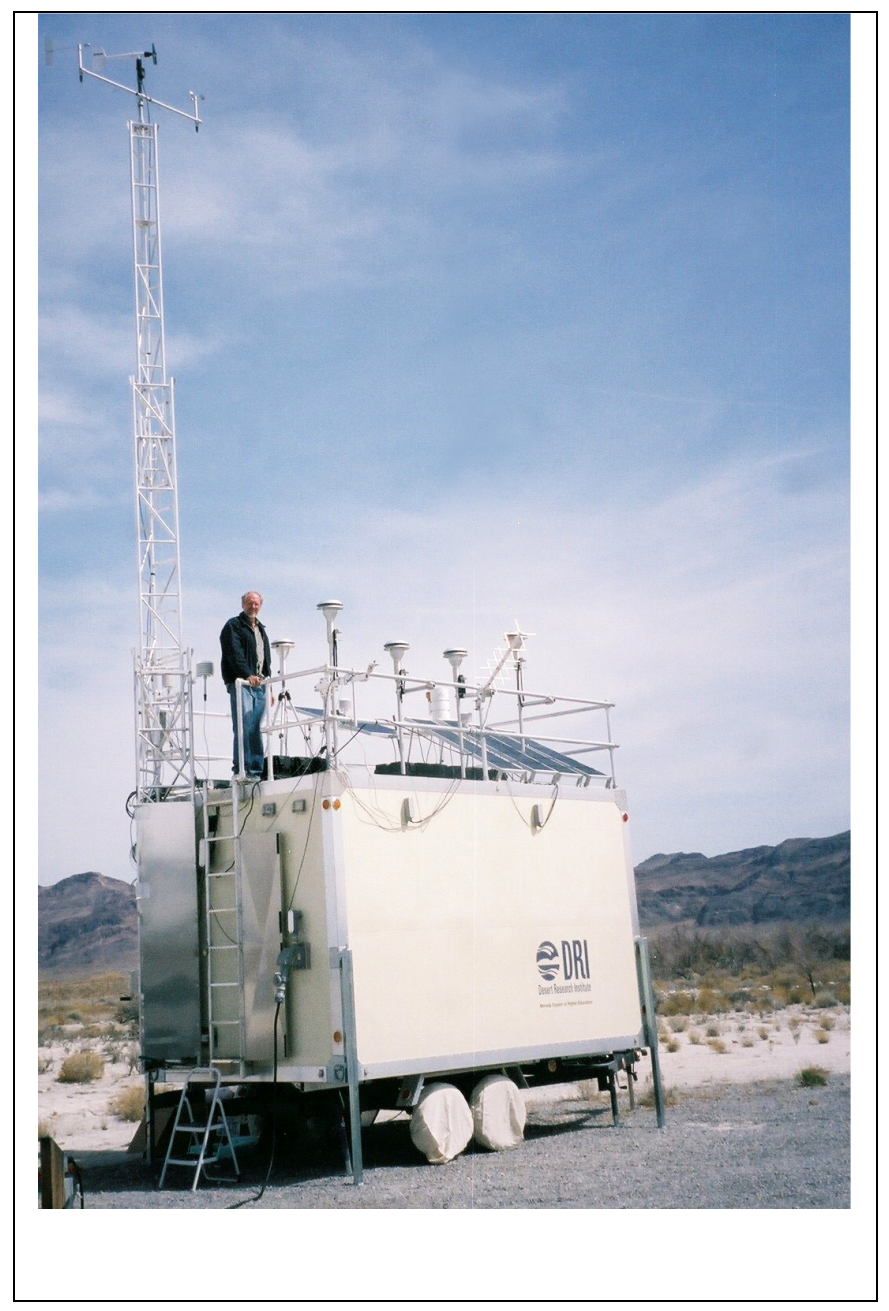

Figure 3. Instrumented mobile shelter at Ash Meadows NWR, showing the meteorological tower with instruments, aerosol sampling inlets, and solar panels.

\section{Aerosol Monitors}

Two Tapered Element Oscillating Microbalance $\left(\mathrm{TEOM}^{\circledR}\right)$ continuous particulate monitors, one each for $\mathrm{PM}_{10}$ (particulate matter $<10 \mu \mathrm{m}$ in aerodynamic diameter) and $\mathrm{PM}_{2.5}$ (particulate matter $<2.5 \mu \mathrm{m}$ in aerodynamic diameter) (Figure 4) were operated at both sites. The TEOM ${ }^{\circledR}$ s were configured to continuously record particulate mass (PM) concentrations, averaged over 15 minute intervals. To validate data from the TEOM ${ }^{\circledR}$ continuous monitors, comparisons were made with results from the BGI PQ100 ${ }^{\circledR}\left(\mathrm{PM}_{10}\right)$ and PQ200 ${ }^{\circledR}\left(\mathrm{PM}_{2.5}\right), 24 \mathrm{hr}$ filter-based samplers. 


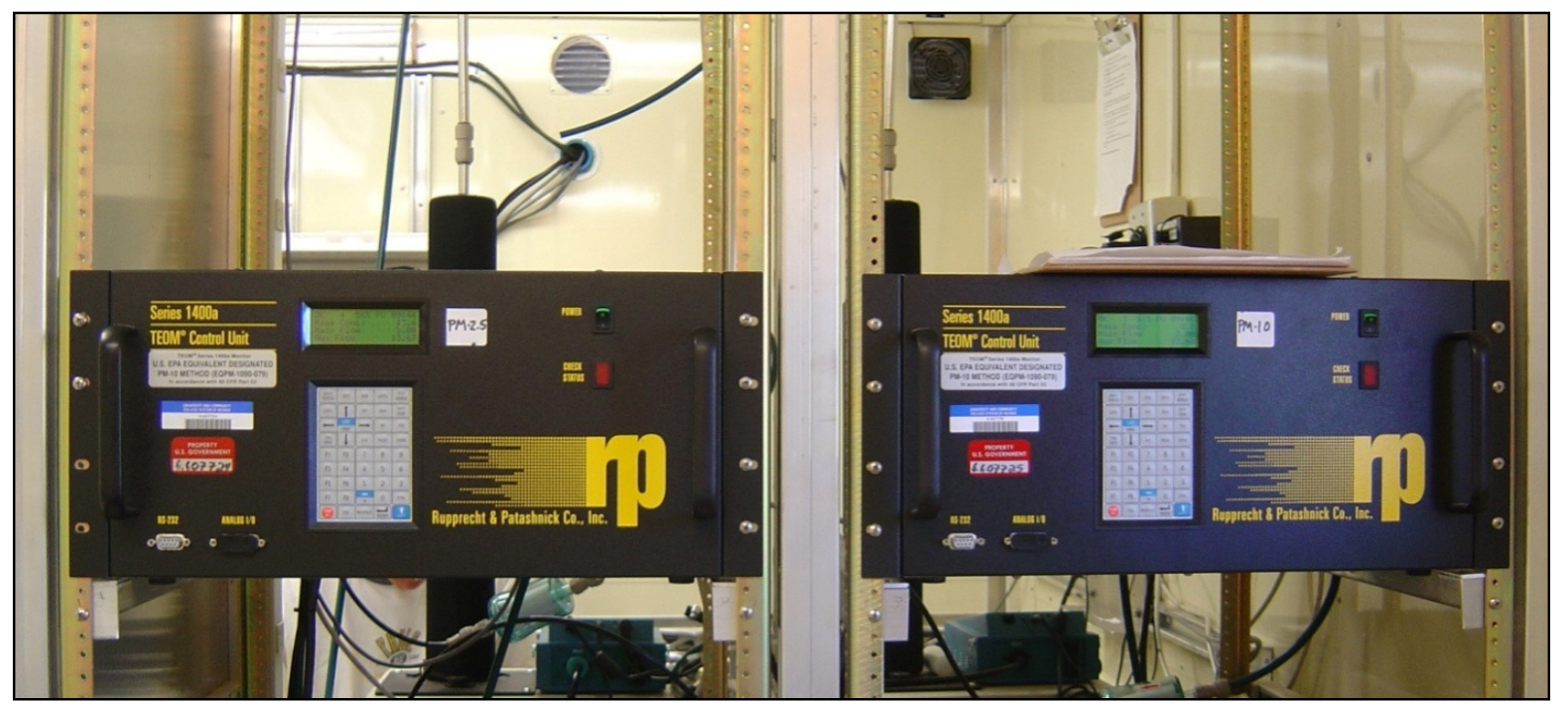

Figure 4. Two rack mounted TEOM ${ }^{\circledR}$ continuous particulate matter (PM) monitors in the mobile shelter, $\mathrm{PM}_{2.5}$ to the left, $\mathrm{PM}_{10}$ to the right.

\section{Filter Samplers}

BGI, Inc., PQ100 ${ }^{\circledR}$, and PQ200 ${ }^{\circledR}$ Ambient PM 2.5 Federal Reference Method (FRM) samplers (Figure 5) collected $24 \mathrm{hr}$ integrated $\mathrm{PM}_{10}$ and $\mathrm{PM}_{2.5}$ aerosol filter samples. Both PQ100 ${ }^{\circledR}$ (Designation No. RFPS-1298-124) and PQ200 ${ }^{\circledR}$ (Designation No. RFPS-0498-116) samplers met the U.S. EPA criteria for collecting $24 \mathrm{hr}$ ambient samples. The $\mathrm{PM}_{10}$ fraction was collected by a filter located downstream of the size selective inlet. For the collection of $\mathrm{PM}_{2.5}$, particles in the range between 2.5 and $10 \mu \mathrm{m}$ were removed by the Very Sharp Cut Cyclone (VSCC) (U.S. Environmental Protection Agency [EPA] Equivalent Designation No. EQPM0202-142), before collection of $\mathrm{PM}_{2.5}$ particles on Teflon ${ }^{\circledR}$ membrane or quartz fiber filters. 


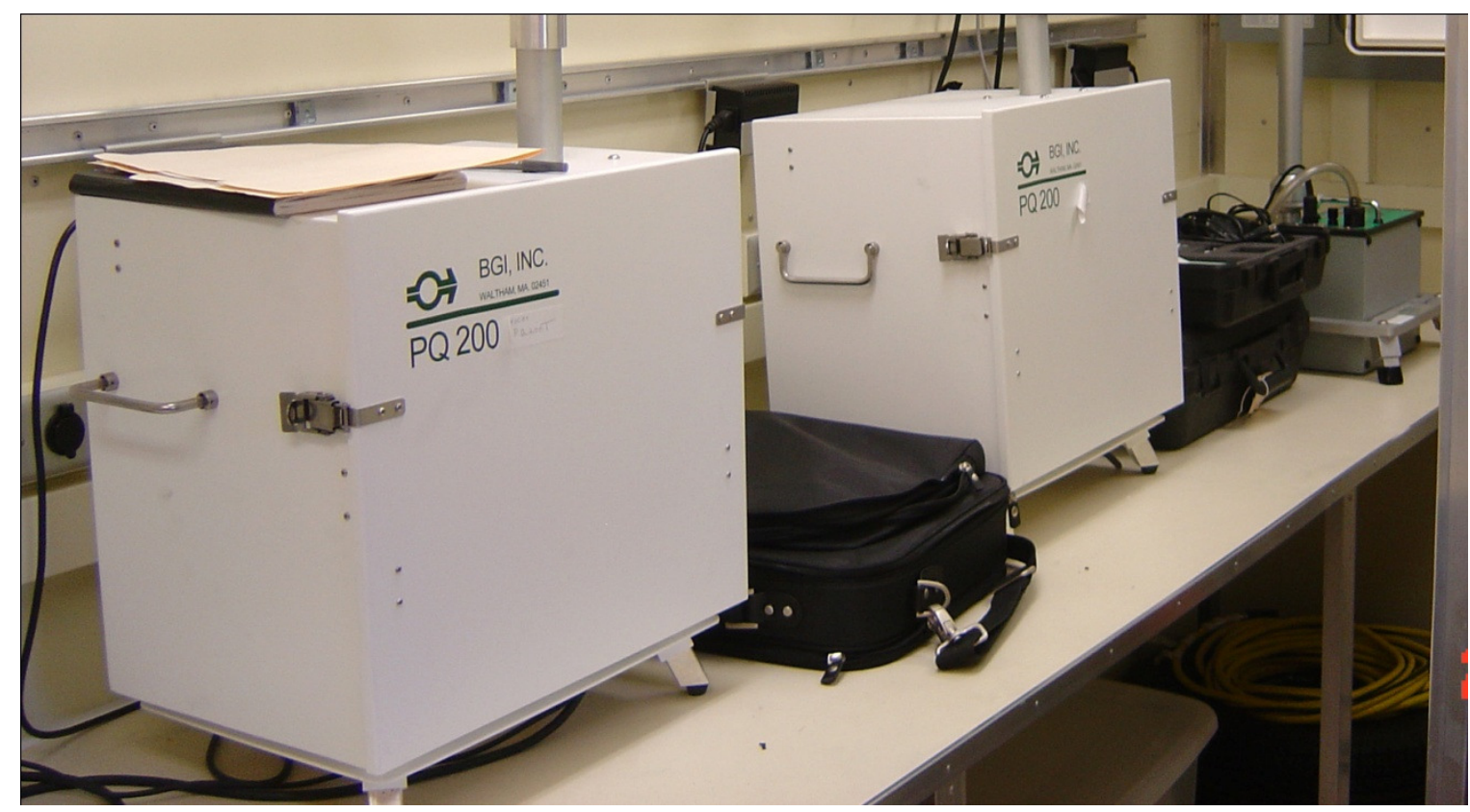

Figure 5. Two BGI PQ200 ${ }^{\circledR}\left(\mathrm{PM}_{2.5}\right)$ (foreground), and one BGI PQ100 ${ }^{\circledR}\left(\mathrm{PM}_{10}\right)$ (green top box in far background) filter samplers mounted on a counter top in the mobile shelter.

Aerosol samples were collected on a US EPA 1-in-12-day sampling schedule. For both PQ100 ${ }^{\circledR}$ and PQ200 ${ }^{\circledR}$ samplers, aerosols were collected at a volumetric flow rate of 16.67 liters per minute $(1 / \mathrm{min})$. The samplers were set up to operate from internal $12 \mathrm{~V} \mathrm{DC}$ batteries, charged by solar panels or from $120 \mathrm{~V}$ AC. Sets of PQ100 ${ }^{\circledR}$ and PQ200 ${ }^{\circledR}$ samplers collected $\mathrm{PM}_{10}$ and $\mathrm{PM}_{2.5}$ aerosol samples, respectively. The Teflon ${ }^{\circledR}$ membrane filters collected particulate matter for gravimetric analysis, light absorption by densitometry, and elemental analysis by X-ray fluorescence (XRF) spectrometry [Watson et al. 1999]. Quartz fiber filters were analyzed for water-soluble ions by atomic absorption spectrometry (AA), ion chromatography (IC), and automated colorimetry (AC) [Chow and Watson 1999], and also for measurement of carbon species by thermal optical reflectance (TOR) [Chow et al. 1993]. The flow rates were calibrated against a BGI Tri-Cal ${ }^{\circledR}$ National Institute of Technology traceable standard. 


\section{Meteorological Equipment}

The datalogger recorded wind speed and direction, precipitation, temperature, humidity and solar radiation at 15 minute intervals. The power for the meteorological instrumentation on the shelter was from $12 \mathrm{~V} \mathrm{DC}$ batteries, charged by a solar panel.

\section{Data Capture}

Continuous data from the air quality and meteorological monitors were stored on a Campbell CR $1000^{\circledR}$ data logger. The measurements from the data logger were automatically polled and relayed to the WRCC at DRI in Reno. This occurs every three hours via the GOES satellite communication system. In addition, data stored in the data logger were routinely downloaded onto a laptop computer. A list of the instrumentation is presented in Table 2.

Table 2. Equipment installed on or inside the mobile air quality shelter

\begin{tabular}{|c|c|c|c|}
\hline CATEGORY & TYPE & INSTRUMENT & SUPPLIER \\
\hline Shelter & Mobile unit & EKTO customized & EKTO \\
\hline \multirow[t]{4}{*}{ Air Quality } & $\begin{array}{l}\text { Filter based } \mathrm{PM}_{10} \\
\text { ( } 2 \text { of each plus one spare) }\end{array}$ & $\begin{array}{l}\text { PQ100 }{ }^{\circledR} \text { - U.S. EPA Federal } \\
\text { Reference Method }\end{array}$ & BGI \\
\hline & $\begin{array}{l}\text { Filter based } \mathrm{PM}_{2.5} \\
\text { ( } 2 \text { of each plus one spare) }\end{array}$ & $\begin{array}{l}\text { PQ200 }{ }^{\circledR}-\text { U.S. EPA Federal } \\
\text { Reference Method }\end{array}$ & BGI \\
\hline & $\begin{array}{l}\text { Continuous } \mathrm{PM}_{10} \\
\text { (1 of each) }\end{array}$ & $\begin{array}{l}\text { TEOM }^{\circledR} \text { Series } 1400 A \text { ambient } \\
\text { particulate monitor - U.S. EPA } \\
\text { equivalency approval (EQPM- } \\
1090-079)\end{array}$ & Thermo Electron \\
\hline & $\begin{array}{l}\text { Continuous } \mathrm{PM}_{2.5} \\
\text { (1 of each) }\end{array}$ & $\begin{array}{l}\text { TEOM }^{(B)} \text { Series } 1400 A \text { ambient } \\
\text { particulate monitor - U.S. EPA } \\
\text { acceptable ( } 40 \text { CFR } 58 \text { ) }\end{array}$ & Thermo Electron \\
\hline Calibration & $\begin{array}{l}\text { Air Flow Rate, } \\
\text { Barometric Pressure } \\
\text { Temperature }\end{array}$ & triCal $^{\circledR}$, NIST traceable standard & BGI \\
\hline Meteorological & $\begin{array}{l}\text { Wind speed \& direction } \\
\text { Precipitation } \\
\text { Temperature/RH } \\
\text { Barometric Pressure } \\
\text { Solar Radiation }\end{array}$ & $\begin{array}{l}\text { Met One, RM Young } \\
\text { Met One, Texas Electronics } \\
\text { Met One, Vaisala } \\
\text { Met One, Vaisala } \\
\text { Met One, Li-Cor }\end{array}$ & $\begin{array}{l}\text { Met One, Campbell } \\
\text { Met One, Campbell } \\
\text { Met One, Campbell } \\
\text { Met One, Campbell } \\
\text { Met One, Campbell }\end{array}$ \\
\hline Data Collection & Data acquisition system & Campbell CR1000 ${ }^{\circledR}$ & Campbell \\
\hline Communication & $\begin{array}{l}\text { GOES satellite transmitter } \\
\text { GPS }\end{array}$ & $\begin{array}{l}\text { TX312 HDR } \\
\text { Garmin }\end{array}$ & $\begin{array}{l}\text { Campbell } \\
\text { Campbell }\end{array}$ \\
\hline Off-grid Power & Solar (5 panels) & $4 \times 40 \mathrm{~W}, 1 \times 55 \mathrm{~W}(215 \mathrm{~W}$ total $)$ & BGI, BP Solar \\
\hline
\end{tabular}




\section{RESULTS}

\section{Continuous PM \& Meteorological Monitoring}

Particulate Matter $(\mathrm{PM})$ concentrations were measured by TEOM ${ }^{\circledR}$ monitors. $\mathrm{PM}_{10}$ levels varied on average from $4.6 \mu \mathrm{g} / \mathrm{m}^{3}$ in winter to $10.8 \mu \mathrm{g} / \mathrm{m}^{3}$ in fall, both well below the $24 \mathrm{hr}$ US Environmental Protection Agency National Ambient Air Quality Standard (NAAQS) for PM 10 of $150 \mu \mathrm{g} / \mathrm{m}^{3}$. The average levels for $\mathrm{PM}_{2.5}$ varied from $0.8 \mu \mathrm{g} / \mathrm{m}^{3}$ in winter to $5.2 \mu \mathrm{g} / \mathrm{m}^{3}$, which are also below the $24 \mathrm{hr}$ NAAQS of $35 \mu \mathrm{g} / \mathrm{m}^{3}$, as well as the annual average NAAQS of 15 $\mu \mathrm{g} / \mathrm{m}^{3}$. Due to instrument failure, no $\mathrm{PM}_{10} \mathrm{TEOM}^{\circledR}$ data are available for spring 2010.

Table 3. Average $\mathrm{PM}_{10}$ and $\mathrm{PM}_{2.5}$ gravimetric results measured at Ash Meadows NWR, by TEOM $^{\circledR}$ continuous monitors, as well as US EPA National Ambient Air Quality Standards (NAAQS) for particulate matter.

\begin{tabular}{|c|c|c|c|c|c|c|}
\hline Season & Months & $\begin{array}{c}\text { Date } \\
\text { Start } \\
(\mathbf{m} / \mathbf{d} / \mathbf{y r}) \\
\end{array}$ & $\begin{array}{c}\text { Date } \\
\text { End } \\
(\mathbf{m} / \mathbf{d} / \mathbf{y r}) \\
\end{array}$ & $\begin{array}{l}\text { Avg. (Unc.) } \\
\mathrm{PM}_{10} \\
\mu \mathrm{g} / \mathrm{m}^{3} \\
\end{array}$ & $\begin{array}{c}\text { Avg. (Unc.) } \\
\mathbf{P M}_{2.5} \\
\mu \mathrm{g} / \mathbf{m}^{3} \\
\end{array}$ & $\mathbf{P M}_{2.5} / \mathbf{P M}_{10}$ \\
\hline Summer & Jun, Jul, Aug & 06/01/09 & 08/31/09 & $10.3(17)$ & $5.2(1.5)$ & 0.50 \\
\hline Fall & Sep, Oct, Nov & 09/01/09 & $11 / 30 / 09$ & $10.8(6.8)$ & $4.3(1.2)$ & 0.39 \\
\hline Winter & Dec, Jan, Feb & $12 / 01 / 09$ & $02 / 28 / 10$ & $4.6(1.1)$ & $0.8(0.4)$ & 0.18 \\
\hline Spring & Mar, Apr, May & 03/01/10 & $05 / 31 / 10$ & n.a. & $4.0(1.6)$ & \\
\hline \multicolumn{2}{|c|}{ Ave. (Std. Dev.) } & & & $8.6(3.4)$ & $3.6(1.9)$ & $0.36(0.16)$ \\
\hline US EPA & NAAQS $24 \mathrm{hr}$ & & & 150 & 35 & \\
\hline US EPA & NAAQS annual & & & & 15 & \\
\hline
\end{tabular}

Seasonally averaged particulate matter (PM) and meteorological data are shown in Tables 4 to 7 and seasonal wind roses are shown in Figures 6 to 9. 
Table 4. Statistics of continuous particulate mass (PM) and meteorological monitoring, as well as Teflon filter measurements, for summer 2009.

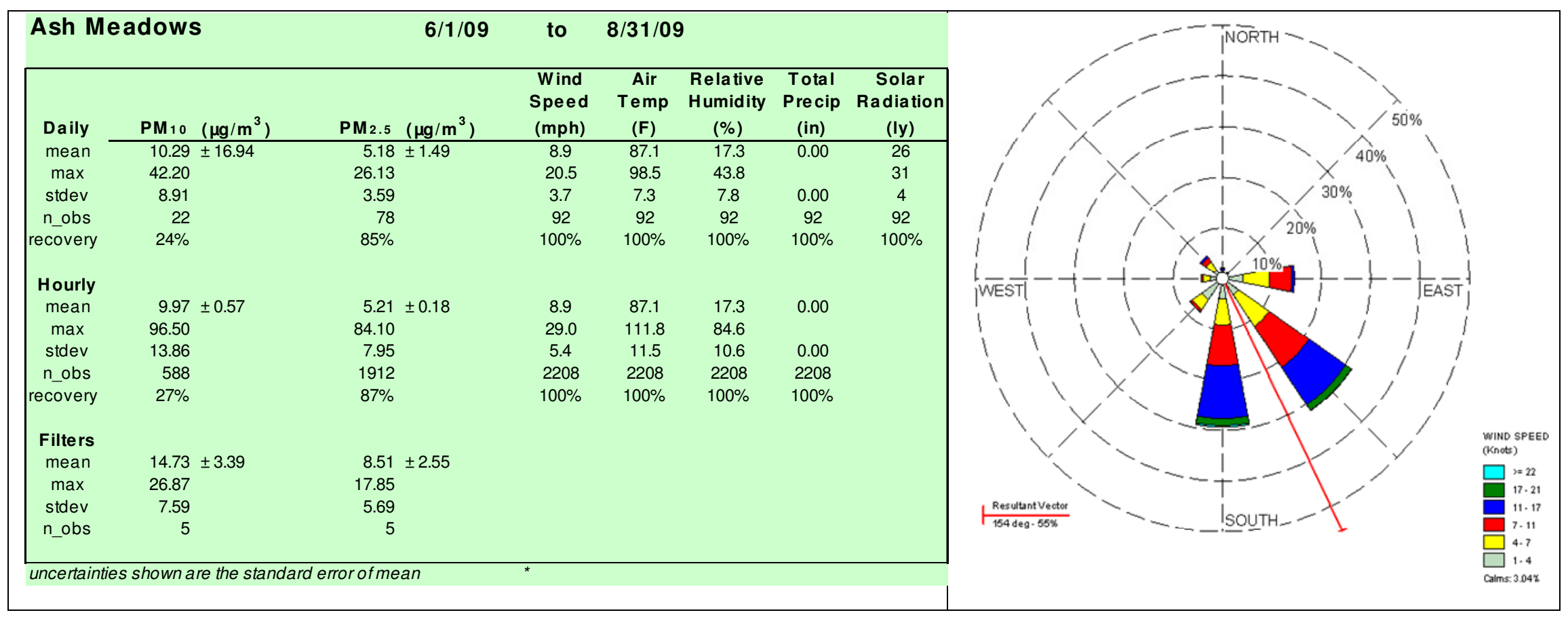

Figure 6. Wind rose shows the predominant wind from the south-southeast for summer 2009. 
Table 5. Statistics of continuous particulate mass (PM) and meteorological monitoring, as well as Teflon filter measurements, for fall 2009.

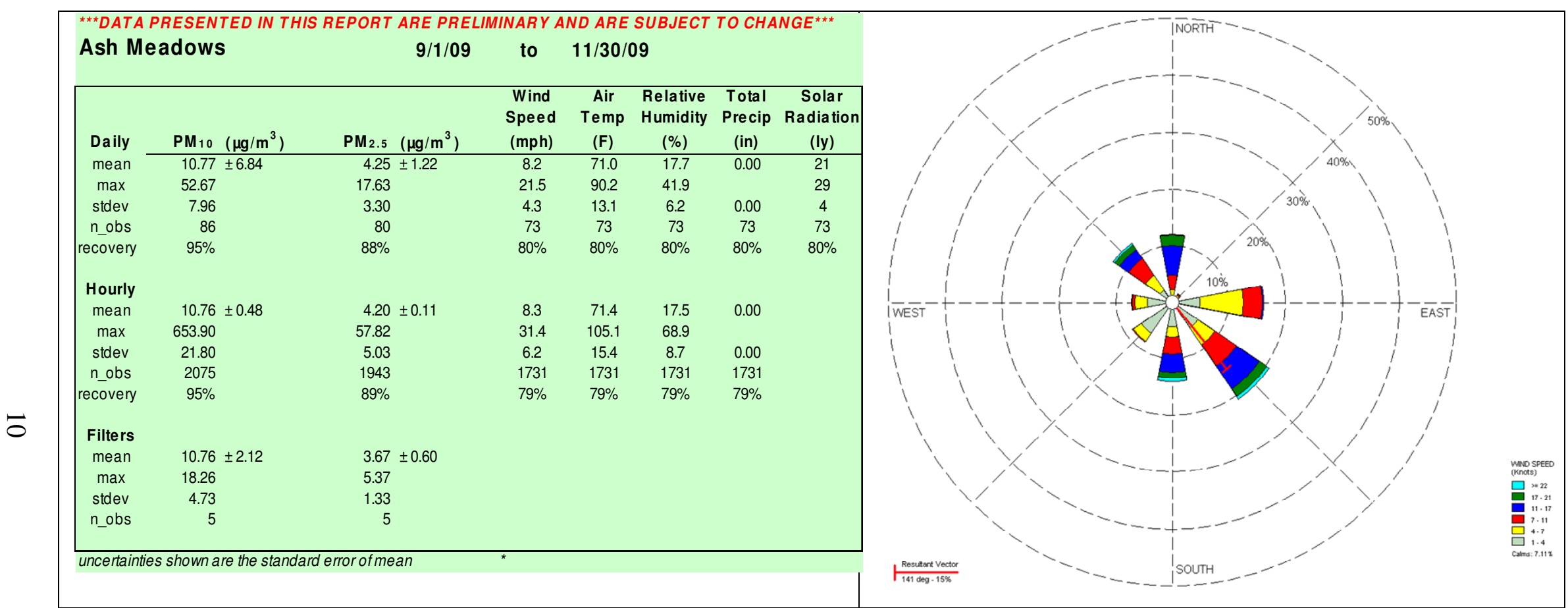

Figure 7. Wind rose shows the predominant wind from the southeast but also with weaker northern to northwesterly and easterly winds in fall 2009. 
Table 6. Statistics of continuous particulate mass (PM) and meteorological monitoring, as well as Teflon filter measurements, for winter 2009/10.

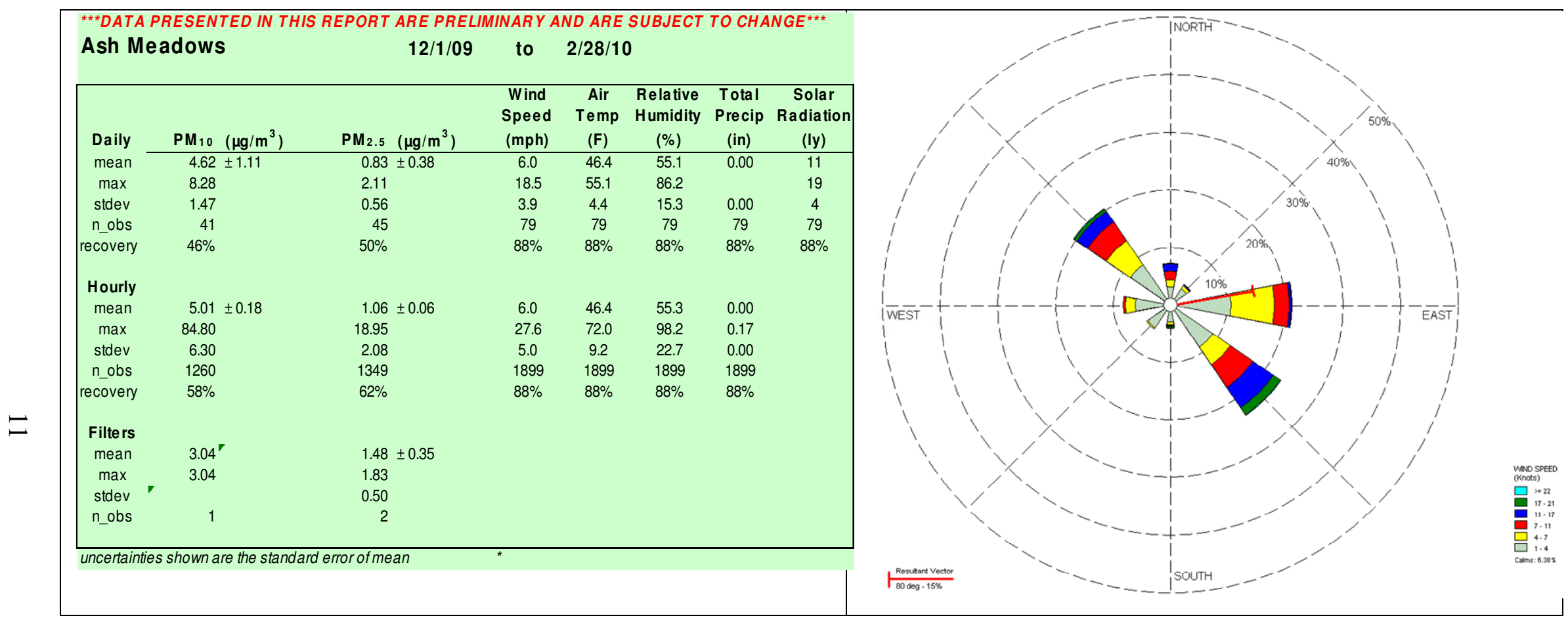

Figure 8. Wind rose shows similar winds from the southeast and northwest, with periods from the east, during winter 2009/10. 
Table 7. Statistics of continuous particulate mass (PM) and meteorological monitoring, as well as Teflon filter measurements, for spring 2010.

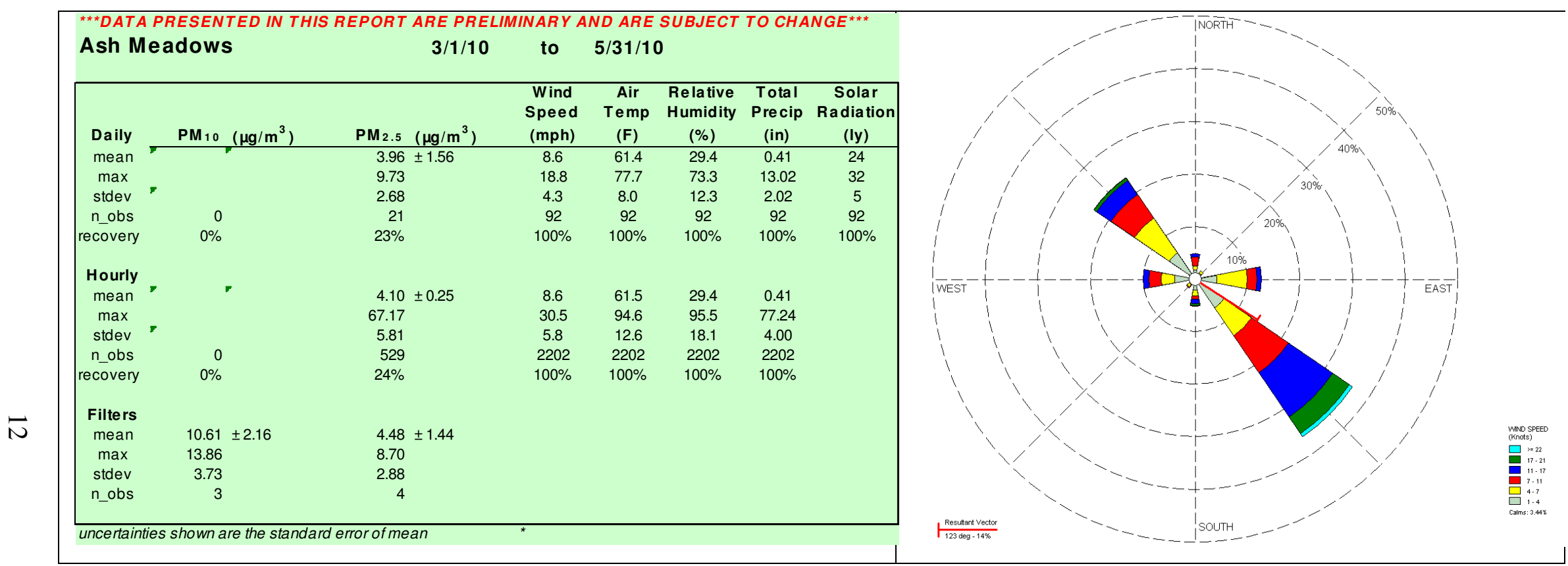

Figure 9. Wind rose shows the predominant wind from the southeast and to a lesser degree from the northwest, for spring 2010. 


\section{Filter Sampling and Analysis}

Fourteen $\mathrm{PM}_{10}$ and $\mathrm{PM}_{2.5}$ filter sample sets collected during the year-long sampling campaign were gravimetrically and chemically analyzed (Tables 8 to 12).

\section{Gravimetric Results}

The particulate mass measured on Teflon ${ }^{\circledR}$ filters varied from $3.0 \mu \mathrm{g} / \mathrm{m}^{3}$ on $12 / 27 / 2009$ to as high as $26.9 \mu \mathrm{g} / \mathrm{m}^{3}$ on $08 / 29 / 2009$ for $\mathrm{PM}_{10}$, and from $1.1 \mu \mathrm{g} / \mathrm{m}^{3}$ to as high as $17.9 \mu \mathrm{g} / \mathrm{m}^{3}$ for $\mathrm{PM}_{2.5}$ on the same two days (Figure 10). The elevated mass values for both $\mathrm{PM}_{10}$ and $\mathrm{PM}_{2.5}$ measured on 08/29/2009 are ascribed to smoke from several wildfires in Los Angeles County, California. These include the Station and Morris fires, both in the Angeles National Forest late in August 2009 (2009http://en.wikipedia.org/wiki/Station_Fire_\%282009\%29\#Los_Angeles_County). The Hysplit backward trajectory model for August 27-29 shows airflow from California towards Ash Meadows NWR for that period (Figure 11).

The $\mathrm{PM}_{2.5} / \mathrm{PM}_{10}$ mass ratios (Table 13) increased from 0.24 on $10 / 28 / 2009$ to 0.66 on $08 / 29 / 09$, the latter indicating that atmospheric aerosol was dominated by smoke. Table 13 shows a comparison of the seasonal average gravimetric results from Ash Meadows NWR to eight urban sites in southwestern Nevada [Engelbrecht et al. 2011], the urban Chemical Speciation Network (CSN) and rural IMPROVE sites in the southwestern U.S. The eight rural sites in Nevada include Ash Meadows NWR [Engelbrecht et al. 2007a], Beatty [Engelbrecht et al. 2007b], Rachel [Engelbrecht et al. 2007c], Sarcobatus Flat [Engelbrecht et al. 2007d], Caliente [Engelbrecht et al. 2008a], Crater Flat [Engelbrecht et al. 2008b], Pahranagat NWR [Engelbrecht et al. 2008c], and Tonopah airport [Engelbrecht et al. 2008d]. The five urban sites are in Las Vegas and Tonopah, Nevada; Los Angeles and Calexico, California; and Phoenix, Arizona, which form part of USEPA's CSN monitoring program. The four rural IMPROVE sites are Domeland Wilderness Area and Joshua Tree National Park (NP) in California, and the Bosque del Apache National Wildlife Refuge (NWR) and Salt Creek Wilderness Area in New Mexico. 
Table 8. Chemical and normative mineral compositions for spring and summer 2009 samples.

\begin{tabular}{|c|c|c|c|c|c|c|c|c|c|c|c|c|}
\hline \multirow{5}{*}{\begin{tabular}{|l} 
SITE \\
TID \\
DATE \\
SIZE \\
\end{tabular}} & \multicolumn{4}{|c|}{ Ash Meadows NWR } & \multicolumn{4}{|c|}{ Ash Meadows NWR } & \multicolumn{4}{|c|}{ Ash Meadows NWR } \\
\hline & \multirow{2}{*}{\multicolumn{4}{|c|}{$\begin{array}{c}\text { YUCTT137 } \\
05 / 25 / 09\end{array}$}} & \multicolumn{4}{|c|}{ YUCTT140 } & \multirow{2}{*}{\multicolumn{4}{|c|}{$\begin{array}{c}\text { YUCTT141 } \\
07 / 25 / 09\end{array}$}} \\
\hline & & & & & & $07 / 1$ & & & & & & \\
\hline & $\underline{\mathrm{PM}}$ & & $\underline{\mathrm{PM}}$ & & $\underline{P N}$ & & $\underline{P M}$ & & $\underline{\mathrm{PN}}$ & & $\underline{\mathrm{PM}}$ & \\
\hline & Conc & Unc & Conc & Unc & Conc & Unc & Conc & Unc & Conc & Unc & Conc & Unc \\
\hline $\mathrm{Cl}^{-}$ & 0.0000 & 0.0210 & 0.0311 & 0.0212 & 0.0185 & 0.0209 & 0.0070 & 0.0208 & 0.0223 & 0.0210 & 0.0000 & 0.0208 \\
\hline $\mathrm{NO}_{3}^{-}$ & 0.4062 & 0.0339 & 0.5590 & 0.0423 & 0.2366 & 0.0260 & 0.1015 & 0.0219 & 0.8208 & 0.0579 & 0.3201 & 0.0296 \\
\hline $\mathrm{SO}_{4}=$ & 1.2646 & 0.0672 & 1.5494 & 0.0810 & 1.1068 & 0.0597 & 0.9997 & 0.0546 & 1.8212 & 0.0944 & 1.5866 & 0.0828 \\
\hline $\mathrm{NH}_{4}^{+}$ & 0.4778 & 0.0336 & 0.5540 & 0.0370 & 0.3076 & 0.0268 & 0.3297 & 0.0276 & 0.5310 & 0.0359 & 0.4828 & 0.0338 \\
\hline $\mathrm{Na}^{+}$ & 0.0936 & 0.0057 & 0.2239 & 0.0133 & 0.1088 & 0.0065 & 0.0580 & 0.0036 & 0.3102 & 0.0183 & 0.2213 & 0.0131 \\
\hline $\mathrm{Mg}^{2+}$ & 0.0351 & 0.0019 & 0.0389 & 0.0021 & 0.0338 & 0.0019 & 0.0101 & 0.0010 & 0.0932 & 0.0048 & 0.0308 & 0.0018 \\
\hline $\mathrm{K}^{+}$ & 0.0744 & 0.0045 & 0.0511 & 0.0034 & 0.0510 & 0.0034 & 0.0272 & 0.0025 & 0.1001 & 0.0057 & 0.0669 & 0.0041 \\
\hline $\mathrm{Ca}^{2+}$ & 0.3185 & 0.0193 & 0.1579 & 0.0132 & 0.3559 & 0.0209 & 0.0836 & 0.0112 & 0.7431 & 0.0393 & 0.1439 & 0.0127 \\
\hline O1TC & 0.5510 & 0.2733 & 0.1298 & 0.0660 & 0.2835 & 0.1412 & 0.1174 & 0.0600 & 0.1924 & 0.0964 & 0.2072 & 0.1037 \\
\hline O2TC & 0.7436 & 0.0495 & 0.6498 & 0.0453 & 0.9663 & 0.0600 & 0.8771 & 0.0557 & 1.3132 & 0.0774 & 0.7357 & 0.0491 \\
\hline O3TC & 0.8694 & 0.1458 & 0.7411 & 0.1332 & 0.8220 & 0.1410 & 0.5767 & 0.1185 & 1.0470 & 0.1645 & 0.5844 & 0.1192 \\
\hline O4TC & 0.4225 & 0.1302 & 0.3637 & 0.1135 & 0.4375 & 0.1344 & 0.2040 & 0.0703 & 0.6808 & 0.2048 & 0.2355 & 0.0785 \\
\hline OPTTC & 0.5736 & 0.1316 & 0.2249 & 0.0569 & 0.4010 & 0.0939 & 0.1829 & 0.0487 & 0.5116 & 0.1179 & 0.2349 & 0.0589 \\
\hline OPTRC & 0.6416 & 0.1443 & 0.2592 & 0.0630 & 0.2443 & 0.0600 & 0.1829 & 0.0482 & 0.5215 & 0.1183 & 0.2542 & 0.0620 \\
\hline $\mathrm{OC}$ & 3.2281 & 0.4241 & 2.1436 & 0.2989 & 2.7535 & 0.3684 & 1.9581 & 0.2784 & 3.7550 & 0.4868 & 2.0170 & 0.2849 \\
\hline E1TC & 0.6449 & 0.0570 & 0.3109 & 0.0324 & 0.3555 & 0.0354 & 0.1829 & 0.0248 & 0.5857 & 0.0524 & 0.2991 & 0.0316 \\
\hline E2TC & 0.1920 & 0.0469 & 0.0928 & 0.0312 & 0.1819 & 0.0451 & 0.0497 & 0.0266 & 0.2850 & 0.0642 & 0.0933 & 0.0313 \\
\hline E3TC & 0.0000 & 0.0082 & 0.0000 & 0.0082 & 0.0000 & 0.0082 & 0.0000 & 0.0082 & 0.0000 & 0.0082 & 0.0000 & 0.0082 \\
\hline EC & 0.1954 & 0.0503 & 0.1445 & 0.0427 & 0.2932 & 0.0670 & 0.0497 & 0.0327 & 0.3491 & 0.0773 & 0.1382 & 0.0418 \\
\hline TC & 3.4235 & 0.4665 & 2.2881 & 0.3317 & 3.0467 & 0.4209 & 2.0078 & 0.3001 & 4.1041 & 0.5501 & 2.1552 & 0.3165 \\
\hline $\mathrm{Na}$ & 0.1087 & 0.0691 & 0.2046 & 0.0723 & 0.0000 & 0.0654 & 0.0513 & 0.0674 & 0.0845 & 0.0683 & 0.0000 & 0.0645 \\
\hline $\mathrm{Mg}$ & 0.0000 & 0.0210 & 0.0000 & 0.0211 & 0.0000 & 0.0210 & 0.0000 & 0.0211 & 0.0000 & 0.0211 & 0.0000 & 0.0210 \\
\hline Al & 0.3654 & 0.0192 & 0.1263 & 0.0079 & 0.0997 & 0.0068 & 0.0417 & 0.0049 & 0.4924 & 0.0254 & 0.1670 & 0.0097 \\
\hline $\mathrm{Si}$ & 1.1828 & 0.0596 & 0.3445 & 0.0177 & 0.4480 & 0.0228 & 0.1134 & 0.0064 & 1.5171 & 0.0764 & 0.4385 & 0.0223 \\
\hline$S$ & 0.6519 & 0.0327 & 0.6541 & 0.0328 & 0.4212 & 0.0212 & 0.3877 & 0.0195 & 0.7003 & 0.0351 & 0.6225 & 0.0312 \\
\hline $\mathrm{Cl}$ & 0.0238 & 0.0014 & 0.0076 & 0.0008 & 0.0087 & 0.0009 & 0.0021 & 0.0007 & 0.0279 & 0.0016 & 0.0079 & 0.0008 \\
\hline K & 0.1892 & 0.0095 & 0.0674 & 0.0035 & 0.0766 & 0.0040 & 0.0306 & 0.0018 & 0.2760 & 0.0139 & 0.1088 & 0.0055 \\
\hline $\mathrm{Ca}$ & 0.3776 & 0.0193 & 0.0641 & 0.0045 & 0.1341 & 0.0075 & 0.0043 & 0.0031 & 0.5611 & 0.0284 & 0.1054 & 0.0062 \\
\hline$\pi$ & 0.0302 & 0.0019 & 0.0097 & 0.0012 & 0.0138 & 0.0013 & 0.0038 & 0.0011 & 0.0416 & 0.0024 & 0.0121 & 0.0012 \\
\hline V & 0.0016 & 0.0007 & 0.0008 & 0.0007 & 0.0000 & 0.0007 & 0.0000 & 0.0007 & 0.0023 & 0.0007 & 0.0000 & 0.0007 \\
\hline $\mathrm{Mn}$ & 0.0053 & 0.0022 & 0.0017 & 0.0022 & 0.0013 & 0.0021 & 0.0000 & 0.0021 & 0.0129 & 0.0023 & 0.0020 & 0.0021 \\
\hline $\mathrm{Fe}$ & 0.3469 & 0.0174 & 0.1021 & 0.0052 & 0.1439 & 0.0073 & 0.0358 & 0.0019 & 0.4497 & 0.0225 & 0.1256 & 0.0063 \\
\hline $\mathrm{Cu}$ & 0.0017 & 0.0025 & 0.0005 & 0.0025 & 0.0014 & 0.0025 & 0.0015 & 0.0025 & 0.0034 & 0.0025 & 0.0015 & 0.0025 \\
\hline$Z n$ & 0.0000 & 0.0007 & 0.0000 & 0.0007 & 0.0010 & 0.0007 & 0.0022 & 0.0007 & 0.0068 & 0.0008 & 0.0015 & 0.0007 \\
\hline $\mathrm{Br}$ & 0.0037 & 0.0007 & 0.0026 & 0.0007 & 0.0001 & 0.0007 & 0.0013 & 0.0007 & 0.0051 & 0.0007 & 0.0036 & 0.0007 \\
\hline $\mathrm{Rb}$ & 0.0010 & 0.0007 & 0.0007 & 0.0007 & 0.0000 & 0.0007 & 0.0000 & 0.0007 & 0.0010 & 0.0007 & 0.0004 & 0.0007 \\
\hline $\mathrm{Sr}$ & 0.0046 & 0.0007 & 0.0007 & 0.0007 & 0.0014 & 0.0007 & 0.0000 & 0.0007 & 0.0044 & 0.0007 & 0.0015 & 0.0007 \\
\hline $\mathrm{Pb}$ & 0.0006 & 0.0008 & 0.0000 & 0.0008 & 0.0000 & 0.0008 & 0.0007 & 0.0008 & 0.0004 & 0.0008 & 0.0020 & 0.0008 \\
\hline Silicate Oxides & & & & & & & & & & & & \\
\hline$\overline{\mathrm{Na}_{2} \mathrm{O}}$ & 0.1262 & & 0.1913 & & 0.1305 & & 0.0719 & & 0.3986 & & 0.1664 & \\
\hline $\mathrm{K}_{2} \mathrm{O}$ & 0.0896 & & 0.0616 & & 0.0615 & & 0.0328 & & 0.1206 & & 0.0806 & \\
\hline $\mathrm{CaO}$ & 0.2663 & & 0.0000 & & 0.2229 & & 0.0000 & & 0.4306 & & 0.0000 & \\
\hline $\mathrm{MgO}$ & 0.0000 & & 0.0000 & & 0.0000 & & 0.0000 & & 0.0000 & & 0.0000 & \\
\hline $\mathrm{Al}_{2} \mathrm{O}_{3}$ & 0.6904 & & 0.2386 & & 0.1884 & & 0.0788 & & 0.9304 & & 0.3156 & \\
\hline $\mathrm{SiO}_{2}$ & 2.5298 & & 0.7368 & & 0.9582 & & 0.2425 & & 3.2448 & & 0.9379 & \\
\hline Silicates & 3.7023 & & 1.2284 & & 1.5614 & & 0.4260 & & 5.1251 & & 1.5005 & \\
\hline Oxides & & & & & & & & & & & & \\
\hline $\mathrm{TiO}_{2}$ & 0.0504 & & 0.0162 & & 0.0230 & & 0.0063 & & 0.0694 & & 0.0202 & \\
\hline $\mathrm{V}_{2} \mathrm{O}_{5}$ & 0.0057 & & 0.0029 & & 0.0000 & & 0.0000 & & 0.0082 & & 0.0000 & \\
\hline $\mathrm{MnO}_{2}$ & 0.0084 & & 0.0027 & & 0.0021 & & 0.0000 & & 0.0204 & & 0.0032 & \\
\hline $\mathrm{Fe}_{2} \mathrm{O}_{3}$ & 0.4960 & & 0.1460 & & 0.2057 & & 0.0512 & & 0.6429 & & 0.1796 & \\
\hline Oxides & 0.5604 & & 0.1677 & & 0.2308 & & 0.0575 & & 0.7410 & & 0.2029 & \\
\hline & & & & & & & & & & & & \\
\hline lonic Compounds & & & & & & & & & & & & \\
\hline $\mathrm{NH}_{4} \mathrm{NO}_{3}$ & 0.5244 & & 0.7216 & & 0.3054 & & 0.1310 & & 1.0596 & & 0.4132 & \\
\hline$\left(\mathrm{NH}_{4}\right)_{2} \mathrm{SO}_{4}$ & 1.3170 & & 1.4332 & & 0.8744 & & 1.0993 & & 1.0700 & & 1.4270 & \\
\hline $\mathrm{CaSO}_{4 .} 2\left(\mathrm{H}_{2} \mathrm{O}\right)$ & 0.5506 & & 0.6782 & & 0.8444 & & 0.3591 & & 1.8697 & & 0.6180 & \\
\hline $\mathrm{NaCl}$ & 0.0000 & & 0.0513 & & 0.0305 & & 0.0115 & & 0.0368 & & 0.0000 & \\
\hline $\mathrm{Na}_{2} \mathrm{SO}_{4}$ & 0.0000 & & 0.1909 & & 0.0000 & & 0.0004 & & 0.0000 & & 0.3022 & \\
\hline $\mathrm{K}_{2} \mathrm{SO}_{4}$ & 0.0000 & & 0.0000 & & 0.0000 & & 0.0000 & & 0.0000 & & 0.0000 & \\
\hline Carbon & & & & & & & & & & & & \\
\hline$\overline{\mathrm{EC}}$ & 0.1954 & & 0.1445 & & 0.2932 & & 0.0497 & & 0.3491 & & 0.1382 & \\
\hline $\mathrm{OC}$ & 3.2281 & & 2.1436 & & 2.7535 & & 1.9581 & & 3.7550 & & 2.0170 & \\
\hline & & & & & & & & & & & & \\
\hline Sum of Species & 10.0782 & & 6.7594 & & 6.8936 & & 4.0926 & & 14.0063 & & 6.6191 & \\
\hline Measured Mass & 15.3078 & 0.7826 & 7.3719 & 0.4033 & 7.2795 & 0.3990 & 3.9967 & 0.2582 & 16.1398 & 0.8234 & 7.1577 & 0.3935 \\
\hline $\mathrm{PM}_{2.5} / \mathrm{PM}_{10}$ & & 0. & & & & 0. & & & & 0. & & \\
\hline
\end{tabular}


Table 9. Chemical and normative mineral compositions for summer and fall 2009 samples.

\begin{tabular}{|c|c|c|c|c|c|c|c|c|c|c|c|c|}
\hline \multirow{5}{*}{\begin{tabular}{|l} 
SITE \\
TID \\
DATE \\
SIZE \\
\end{tabular}} & \multicolumn{4}{|c|}{ Ash Meadows NWR } & \multicolumn{4}{|c|}{ Ash Meadows NWR } & \multicolumn{4}{|c|}{ Ash Meadows NWR } \\
\hline & \multirow{2}{*}{\multicolumn{4}{|c|}{$\begin{array}{c}\text { YUCTT143 } \\
08 / 05 / 09\end{array}$}} & \multicolumn{4}{|c|}{ YUCTT145 } & \multicolumn{4}{|c|}{ YUCTT146 } \\
\hline & & & & & & $08 / 2$ & & & & $09 / 1$ & & \\
\hline & $\underline{P M}$ & & PM & & $\underline{\mathrm{PN}}$ & & $\underline{P N}$ & & $\underline{P N}$ & & $\underline{\mathrm{PN}}$ & \\
\hline & Conc & Unc & Conc & Unc & Conc & Unc & Conc & Unc & Conc & Unc & Conc & Unc \\
\hline $\mathrm{Cl}^{-}$ & 0.0000 & 0.0208 & 0.0000 & 0.0211 & 0.0065 & 0.0208 & 0.0069 & 0.0208 & 0.0772 & 0.0231 & 0.0000 & 0.0210 \\
\hline $\mathrm{NO}_{3}^{-}$ & 0.4833 & 0.0380 & 0.1416 & 0.0228 & 0.8562 & 0.0601 & 0.5986 & 0.0446 & 0.7017 & 0.0507 & 0.2475 & 0.0264 \\
\hline $\mathrm{SO}_{4}{ }^{\mathrm{I}}$ & 1.2281 & 0.0655 & 1.0289 & 0.0560 & 1.3686 & 0.0722 & 1.3007 & 0.0689 & 0.9121 & 0.0506 & 0.7865 & 0.0449 \\
\hline $\mathrm{NH}_{4}^{+}$ & 0.3856 & 0.0297 & 0.3187 & 0.0272 & 0.4778 & 0.0336 & 0.4658 & 0.0331 & 0.2537 & 0.0251 & 0.2362 & 0.0246 \\
\hline $\mathrm{Na}^{+}$ & 0.1537 & 0.0092 & 0.0636 & 0.0040 & 0.1146 & 0.0069 & 0.0773 & 0.0047 & 0.2611 & 0.0155 & 0.1172 & 0.0070 \\
\hline $\mathrm{Mg}^{2+}$ & 0.0454 & 0.0024 & 0.0120 & 0.0010 & 0.1166 & 0.0059 & 0.0267 & 0.0016 & 0.1268 & 0.0064 & 0.0297 & 0.0017 \\
\hline $\mathrm{K}^{+}$ & 0.0485 & 0.0033 & 0.0201 & 0.0023 & 0.4926 & 0.0263 & 0.4422 & 0.0236 & 0.0865 & 0.0050 & 0.0378 & 0.0029 \\
\hline $\mathrm{Ca}^{2+}$ & 0.4546 & 0.0254 & 0.0716 & 0.0110 & -99 & -99 & 0.2443 & 0.0162 & 1.3092 & 0.0675 & 0.2103 & 0.0149 \\
\hline O1TC & 0.0988 & 0.0511 & 0.0504 & 0.0290 & 0.3049 & 0.1517 & 0.1891 & 0.0948 & 0.1905 & 0.0955 & 0.0079 & 0.0152 \\
\hline O2TC & 0.9712 & 0.0602 & 0.6129 & 0.0437 & 1.4604 & 0.0850 & 1.8235 & 0.1041 & 0.7130 & 0.0481 & 0.7186 & 0.0483 \\
\hline О3TC & 0.8294 & 0.1418 & 0.4120 & 0.1062 & 3.0621 & 0.4103 & 2.7445 & 0.3700 & 0.7532 & 0.1343 & 0.5821 & 0.1190 \\
\hline O4TC & 0.4058 & 0.1254 & 0.1203 & 0.0506 & 2.1202 & 0.6288 & 1.1348 & 0.3380 & 0.4324 & 0.1330 & 0.2794 & 0.0902 \\
\hline OPTTC & 0.3936 & 0.0923 & 0.0771 & 0.0314 & 1.8191 & 0.4099 & 1.9093 & 0.4301 & 0.3208 & 0.0767 & 0.2005 & 0.0521 \\
\hline OPTRC & 0.2033 & 0.0520 & 0.0530 & 0.0286 & 1.5216 & 0.3375 & 1.6065 & 0.3562 & 0.2162 & 0.0545 & 0.1378 & 0.0401 \\
\hline OC & 2.5085 & 0.3402 & 1.2485 & 0.2054 & 8.4691 & 1.0641 & 7.4984 & 0.9442 & 2.3054 & 0.3170 & 1.7258 & 0.2534 \\
\hline E1TC & 0.3321 & 0.0338 & 0.0771 & 0.0206 & 2.4411 & 0.2033 & 2.4500 & 0.2041 & 0.3208 & 0.0330 & 0.2005 & 0.0257 \\
\hline E2TC & 0.1620 & 0.0417 & 0.0324 & 0.0254 & 0.2846 & 0.0641 & 0.1955 & 0.0475 & 0.1771 & 0.0443 & 0.1186 & 0.0348 \\
\hline E3TC & 0.0000 & 0.0082 & 0.0000 & 0.0082 & 0.0000 & 0.0082 & 0.0000 & 0.0082 & 0.0000 & 0.0082 & 0.0000 & 0.0082 \\
\hline ECTRC & 0.2908 & 0.0666 & 0.0565 & 0.0331 & 1.2040 & 0.2460 & 1.0390 & 0.2129 & 0.2817 & 0.0650 & 0.1812 & 0.0481 \\
\hline TC & 2.7993 & 0.3913 & 1.3051 & 0.2267 & 9.6732 & 1.2555 & 8.5375 & 1.1104 & 2.5871 & 0.3663 & 1.9070 & 0.2889 \\
\hline $\mathrm{Na}$ & 0.0000 & 0.0656 & 0.0000 & 0.0641 & 0.0940 & 0.0686 & 0.0000 & 0.0659 & 0.0000 & 0.0644 & 0.0000 & 0.0651 \\
\hline $\mathrm{Mg}$ & 0.0000 & 0.0208 & 0.0000 & 0.0210 & 0.0183 & 0.0214 & 0.0000 & 0.0211 & 0.0182 & 0.0214 & 0.0000 & 0.0210 \\
\hline $\mathrm{Al}$ & 0.2930 & 0.0156 & 0.0890 & 0.0064 & 0.3870 & 0.0202 & 0.1223 & 0.0078 & 0.2154 & 0.0119 & 0.1298 & 0.0081 \\
\hline $\mathrm{Si}$ & 0.8253 & 0.0417 & 0.2000 & 0.0106 & 1.0429 & 0.0526 & 0.2118 & 0.0111 & 0.8713 & 0.0440 & 0.2091 & 0.0110 \\
\hline S & 0.4746 & 0.0238 & 0.4075 & 0.0205 & 0.5517 & 0.0277 & 0.5123 & 0.0257 & 0.3236 & 0.0163 & 0.3158 & 0.0159 \\
\hline $\mathrm{Cl}$ & 0.0133 & 0.0010 & 0.0000 & 0.0007 & 0.0293 & 0.0017 & 0.0173 & 0.0011 & 0.0284 & 0.0016 & 0.0053 & 0.0008 \\
\hline K & 0.1285 & 0.0065 & 0.0373 & 0.0021 & 0.6637 & 0.0332 & 0.4582 & 0.0230 & 0.1725 & 0.0087 & 0.0559 & 0.0030 \\
\hline $\mathrm{Ca}$ & 0.2660 & 0.0138 & 0.0305 & 0.0035 & 1.1037 & 0.0555 & 0.1464 & 0.0081 & 1.0652 & 0.0536 & 0.2088 & 0.0110 \\
\hline$\pi$ & 0.0228 & 0.0016 & 0.0055 & 0.0011 & 0.0337 & 0.0020 & 0.0057 & 0.0011 & 0.0192 & 0.0014 & 0.0051 & 0.0011 \\
\hline V & 0.0009 & 0.0007 & 0.0000 & 0.0007 & 0.0012 & 0.0007 & 0.0000 & 0.0007 & 0.0008 & 0.0007 & 0.0001 & 0.0007 \\
\hline$M n$ & 0.0038 & 0.0022 & 0.0000 & 0.0021 & 0.0167 & 0.0023 & 0.0026 & 0.0022 & 0.0038 & 0.0022 & 0.0018 & 0.0021 \\
\hline $\mathrm{Fe}$ & 0.2568 & 0.0129 & 0.0631 & 0.0033 & 0.3387 & 0.0170 & 0.0626 & 0.0032 & 0.2032 & 0.0102 & 0.0512 & 0.0027 \\
\hline $\mathrm{Cu}$ & 0.0024 & 0.0025 & 0.0010 & 0.0025 & 0.0053 & 0.0025 & 0.0009 & 0.0025 & 0.0025 & 0.0025 & 0.0023 & 0.0025 \\
\hline$Z n$ & 0.0003 & 0.0007 & 0.0000 & 0.0007 & 0.0082 & 0.0008 & 0.0075 & 0.0008 & 0.0000 & 0.0007 & 0.0013 & 0.0007 \\
\hline $\mathrm{Br}$ & 0.0012 & 0.0007 & 0.0014 & 0.0007 & 0.0053 & 0.0007 & 0.0061 & 0.0008 & 0.0044 & 0.0007 & 0.0045 & 0.0007 \\
\hline $\mathrm{Rb}$ & 0.0005 & 0.0007 & 0.0000 & 0.0007 & 0.0014 & 0.0007 & 0.0002 & 0.0007 & 0.0007 & 0.0007 & 0.0000 & 0.0007 \\
\hline $\mathrm{Sr}$ & 0.0015 & 0.0007 & 0.0000 & 0.0007 & 0.0125 & 0.0010 & 0.0021 & 0.0007 & 0.0091 & 0.0009 & 0.0006 & 0.0007 \\
\hline $\mathrm{Pb}$ & 0.0000 & 0.0008 & 0.0000 & 0.0008 & 0.0009 & 0.0008 & 0.0000 & 0.0008 & 0.0007 & 0.0008 & 0.0001 & 0.0008 \\
\hline Silicate Oxides & & & & & & & & & & & & \\
\hline $\mathrm{Na}_{2} \mathrm{O}$ & 0.2072 & & 0.0092 & & 0.0000 & & 0.0982 & & 0.2845 & & 0.1580 & \\
\hline $\mathrm{K}_{2} \mathrm{O}$ & 0.0584 & & 0.0242 & & 0.0746 & & 0.5328 & & 0.1042 & & 0.0455 & \\
\hline $\mathrm{CaO}$ & 0.2998 & & 0.0000 & & 0.0000 & & 0.0357 & & 1.3763 & & 0.0903 & \\
\hline $\mathrm{MgO}$ & 0.0000 & & 0.0000 & & 0.0303 & & 0.0000 & & 0.0302 & & 0.0000 & \\
\hline $\mathrm{Al}_{2} \mathrm{O}_{3}$ & 0.5536 & & 0.1682 & & 0.7313 & & 0.2311 & & 0.4070 & & 0.2453 & \\
\hline $\mathrm{SiO}_{2}$ & 1.7652 & & 0.4278 & & 2.2306 & & 0.4530 & & 1.8636 & & 0.4472 & \\
\hline Silicates & 2.8843 & & 0.6294 & & 3.0668 & & 1.3508 & & 4.0658 & & 0.9863 & \\
\hline Oxides & & & & & & & & & & & & \\
\hline $\mathrm{TiO}_{2}$ & 0.0380 & & 0.0092 & & 0.0562 & & 0.0095 & & 0.0320 & & 0.0085 & \\
\hline $\mathrm{V}_{2} \mathrm{O}_{5}$ & 0.0032 & & 0.0000 & & 0.0043 & & 0.0000 & & 0.0029 & & 0.0004 & \\
\hline $\mathrm{MnO}_{2}$ & 0.0060 & & 0.0000 & & 0.0264 & & 0.0041 & & 0.0060 & & 0.0028 & \\
\hline $\mathrm{Fe}_{2} \mathrm{O}_{3}$ & 0.3671 & & 0.0902 & & 0.4842 & & 0.0895 & & 0.2905 & & 0.0732 & \\
\hline Oxides & 0.4144 & & 0.0994 & & 0.5712 & & 0.1031 & & 0.3314 & & 0.0849 & \\
\hline Janis Comn & & & & & & & & & & & & \\
\hline $\mathrm{NH}_{4} \mathrm{NO}_{3}$ & 0.6239 & & 0.1828 & & 11053 & & 07708 & & 00050 & & 03195 & \\
\hline$\left(\mathrm{NH}_{4}\right)_{2} \mathrm{SO}_{4}$ & 0.8972 & & 1.0162 & & $\begin{array}{l}1.1053 \\
0.8375\end{array}$ & & 1.0680 & & $\begin{array}{l}0.9059 \\
0.1814\end{array}$ & & $\begin{array}{l}0.3195 \\
0.6013\end{array}$ & \\
\hline $\mathrm{CaSO}_{4} .2\left(\mathrm{H}_{2} \mathrm{O}\right)$ & 1.0321 & & 0.3075 & & -99 & & 0.9396 & & 1.3982 & & 0.6261 & \\
\hline $\mathrm{NaCl}$ & 0.0000 & & 0.0000 & & 0.0107 & & 0.0114 & & 0.1273 & & 0.0000 & \\
\hline $\mathrm{Na}_{2} \mathrm{SO}_{4}$ & 0.0000 & & 0.1753 & & 0.3410 & & 0.0000 & & 0.0000 & & 0.0000 & \\
\hline $\mathrm{K}_{2} \mathrm{SO}_{4}$ & 0.0000 & & 0.0000 & & 0.9599 & & 0.0000 & & 0.0000 & & 0.0000 & \\
\hline & & & & & & & & & & & & \\
\hline Carbon & & & & & & & & & & & & \\
\hline $\mathrm{EC}$ & 0.2908 & & 0.0565 & & 1.2040 & & 1.0390 & & 0.2817 & & 0.1812 & \\
\hline OC & 2.5085 & & 1.2485 & & 8.4691 & & 7.4984 & & 2.3054 & & 1.7258 & \\
\hline & & & & & & & & & & & & \\
\hline Sum of Species & 8.6511 & & 3.7156 & & 16.5654 & & 12.7831 & & 9.5970 & & 4.5251 & \\
\hline Measured Mass & 9.8170 & 0.5173 & 4.1199 & 0.2630 & 26.8719 & 1.3535 & 17.8527 & 0.9075 & 12.3128 & 0.6370 & 4.4528 & 0.2762 \\
\hline $\mathrm{PM}_{2.5} / \mathrm{PM}_{10}$ & & 0.4 & & & & 0.6 & & & & 0.3 & & \\
\hline
\end{tabular}


Table 10. Chemical and normative mineral compositions for fall 2009 samples.

\begin{tabular}{|c|c|c|c|c|c|c|c|c|c|c|c|c|}
\hline \multirow{5}{*}{\begin{tabular}{|l} 
SITE \\
TID \\
DATE \\
SIZE
\end{tabular}} & \multicolumn{4}{|c|}{ Ash Meadows NWR } & \multicolumn{4}{|c|}{ Ash Meadows NWR } & \multicolumn{4}{|c|}{ Ash Meadows NWR } \\
\hline & \multirow{2}{*}{\multicolumn{4}{|c|}{$\begin{array}{c}\text { YUCTT148 } \\
10 / 04 / 09\end{array}$}} & \multirow{2}{*}{\multicolumn{4}{|c|}{$\begin{array}{c}\text { YUCTT149 } \\
10 / 22 / 09\end{array}$}} & & YUCT & & \\
\hline & & & & & & & & & & $10 / 2$ & & \\
\hline & $\underline{P M}$ & & $\underline{P M}$ & & $\underline{P M}$ & & $\underline{\mathrm{PM}_{2}}$ & & $\underline{\mathrm{PM}}$ & & $\underline{P M}$ & \\
\hline & Conc & Unc & Conc & Unc & Conc & Unc & Conc & Unc & Conc & Unc & Conc & Unc \\
\hline $\mathrm{Cl}^{-}$ & 0.5160 & 0.0708 & 0.2379 & 0.0375 & 0.0000 & 0.0208 & 0.0000 & 0.0211 & 0.0059 & 0.0208 & 0.0000 & 0.0210 \\
\hline $\mathrm{NO}_{3}{ }^{-}$ & 0.5866 & 0.0439 & 0.2609 & 0.0270 & 0.0608 & 0.0212 & 0.0265 & 0.0209 & 0.0751 & 0.0214 & 0.0427 & 0.0210 \\
\hline $\mathrm{SO}_{4}=$ & 0.8254 & 0.0466 & 0.5846 & 0.0361 & 0.4103 & 0.0294 & 0.3599 & 0.0276 & 0.2950 & 0.0256 & 0.2453 & 0.0242 \\
\hline $\mathrm{NH}_{4}^{+}$ & 0.2008 & 0.0236 & 0.1617 & 0.0226 & 0.1404 & 0.0222 & 0.1215 & 0.0219 & 0.0941 & 0.0214 & 0.0926 & 0.0214 \\
\hline $\mathrm{Na}^{+}$ & 0.5156 & 0.0304 & 0.2549 & 0.0151 & 0.0344 & 0.0024 & 0.0137 & 0.0015 & 0.0684 & 0.0042 & 0.0190 & 0.0017 \\
\hline $\mathrm{Mg}^{2+}$ & 0.1290 & 0.0065 & 0.0372 & 0.0020 & 0.1133 & 0.0057 & 0.0191 & 0.0013 & 0.0895 & 0.0046 & 0.0105 & 0.0010 \\
\hline $\mathrm{K}^{+}$ & 0.1056 & 0.0060 & 0.0326 & 0.0027 & 0.0376 & 0.0029 & 0.0147 & 0.0022 & 0.0411 & 0.0030 & 0.0195 & 0.0023 \\
\hline $\mathrm{Ca}^{2+}$ & 1.0847 & 0.0562 & 0.1479 & 0.0128 & 1.4478 & 0.0745 & 0.2305 & 0.0157 & 1.5520 & 0.0798 & 0.1741 & 0.0137 \\
\hline O1TC & 0.1654 & 0.0832 & 0.0540 & 0.0305 & 0.0465 & 0.0273 & 0.0700 & 0.0377 & 0.0624 & 0.0343 & 0.1411 & 0.0714 \\
\hline O2TC & 0.5820 & 0.0424 & 0.3364 & 0.0334 & 0.2287 & 0.0305 & 0.2316 & 0.0306 & 0.2067 & 0.0300 & 0.2745 & 0.0316 \\
\hline O3TC & 0.8377 & 0.1426 & 0.3333 & 0.1014 & 0.3336 & 0.1013 & 0.2981 & 0.0995 & 0.6078 & 0.1211 & 0.6393 & 0.1238 \\
\hline O4TC & 0.4797 & 0.1465 & 0.0659 & 0.0409 & 0.2189 & 0.0741 & 0.0922 & 0.0451 & 0.4194 & 0.1293 & 0.1873 & 0.0661 \\
\hline OPTTC & 0.1654 & 0.0455 & 0.0127 & 0.0263 & 0.1589 & 0.0443 & 0.0629 & 0.0297 & 0.1283 & 0.0389 & 0.0000 & 0.0261 \\
\hline OPTRC & 0.2812 & 0.0675 & 0.0032 & 0.0261 & 0.0937 & 0.0334 & 0.0222 & 0.0266 & 0.2615 & 0.0635 & 0.0470 & 0.0281 \\
\hline $\mathrm{OC}$ & 2.3461 & 0.3216 & 0.7927 & 0.1665 & 0.9213 & 0.1764 & 0.7141 & 0.1609 & 1.5578 & 0.2359 & 1.2892 & 0.2092 \\
\hline E1TC & 0.2812 & 0.0305 & 0.0111 & 0.0197 & 0.1050 & 0.0215 & 0.0272 & 0.0197 & 0.2732 & 0.0300 & 0.0902 & 0.0210 \\
\hline E2TC & 0.1173 & 0.0346 & 0.0016 & 0.0245 & 0.0540 & 0.0270 & 0.0357 & 0.0256 & 0.1186 & 0.0348 & 0.0243 & 0.0250 \\
\hline E3TC & 0.0000 & 0.0082 & 0.0000 & 0.0082 & 0.0000 & 0.0082 & 0.0000 & 0.0082 & 0.0000 & 0.0082 & 0.0000 & 0.0082 \\
\hline ECTRC & 0.1173 & 0.0391 & 0.0095 & 0.0311 & 0.0652 & 0.0337 & 0.0407 & 0.0321 & 0.1302 & 0.0407 & 0.0675 & 0.0339 \\
\hline TC & 2.4634 & 0.3518 & 0.8022 & 0.1838 & 0.9866 & 0.1980 & 0.7549 & 0.1804 & 1.6881 & 0.2653 & 1.3567 & 0.2316 \\
\hline $\mathrm{Na}$ & 0.1875 & 0.0716 & 0.1976 & 0.0720 & 0.0000 & 0.0642 & 0.0000 & 0.0642 & 0.0000 & 0.0635 & 0.0000 & 0.0656 \\
\hline Mg & 0.0000 & 0.0210 & 0.0000 & 0.0211 & 0.0066 & 0.0213 & 0.0000 & 0.0212 & 0.0000 & 0.0210 & 0.0000 & 0.0212 \\
\hline $\mathrm{Al}$ & 0.3180 & 0.0169 & 0.1205 & 0.0077 & 0.1217 & 0.0077 & 0.0223 & 0.0045 & 0.1409 & 0.0085 & 0.0505 & 0.0051 \\
\hline $\mathrm{Si}$ & 1.0207 & 0.0515 & 0.4164 & 0.0212 & 0.5289 & 0.0269 & 0.1607 & 0.0086 & 0.7923 & 0.0400 & 0.1540 & 0.0083 \\
\hline$S$ & 0.2396 & 0.0121 & 0.2413 & 0.0121 & 0.1567 & 0.0079 & 0.1527 & 0.0077 & 0.1057 & 0.0054 & 0.1001 & 0.0051 \\
\hline $\mathrm{Cl}$ & 0.3474 & 0.0175 & 0.2159 & 0.0109 & 0.0015 & 0.0007 & 0.0051 & 0.0008 & 0.0185 & 0.0012 & 0.0035 & 0.0007 \\
\hline K & 0.1553 & 0.0079 & 0.0711 & 0.0037 & 0.0842 & 0.0043 & 0.0267 & 0.0017 & 0.1206 & 0.0061 & 0.0379 & 0.0021 \\
\hline $\mathrm{Ca}$ & 0.3031 & 0.0156 & 0.1133 & 0.0066 & 1.0782 & 0.0542 & 0.2190 & 0.0115 & 1.1446 & 0.0575 & 0.1821 & 0.0097 \\
\hline $\mathrm{Ti}$ & 0.0252 & 0.0017 & 0.0102 & 0.0012 & 0.0076 & 0.0011 & 0.0024 & 0.0010 & 0.0134 & 0.0013 & 0.0029 & 0.0011 \\
\hline V & 0.0001 & 0.0007 & 0.0002 & 0.0007 & 0.0000 & 0.0007 & 0.0002 & 0.0007 & 0.0000 & 0.0007 & 0.0000 & 0.0007 \\
\hline Mn & 0.0075 & 0.0022 & 0.0018 & 0.0022 & 0.0007 & 0.0021 & 0.0000 & 0.0021 & 0.0022 & 0.0021 & 0.0018 & 0.0021 \\
\hline $\mathrm{Fe}$ & 0.2672 & 0.0134 & 0.1030 & 0.0052 & 0.0943 & 0.0048 & 0.0284 & 0.0016 & 0.1494 & 0.0075 & 0.0300 & 0.0017 \\
\hline $\mathrm{Cu}$ & 0.0024 & 0.0025 & 0.0000 & 0.0025 & 0.0000 & 0.0025 & 0.0000 & 0.0025 & 0.0011 & 0.0025 & 0.0002 & 0.0025 \\
\hline $\mathrm{Zn}$ & 0.0000 & 0.0007 & 0.0000 & 0.0007 & 0.0000 & 0.0007 & 0.0000 & 0.0007 & 0.0000 & 0.0007 & 0.0000 & 0.0007 \\
\hline $\mathrm{Br}$ & 0.0016 & 0.0007 & 0.0000 & 0.0007 & 0.0002 & 0.0007 & 0.0000 & 0.0007 & 0.0000 & 0.0007 & 0.0020 & 0.0007 \\
\hline $\mathrm{Rb}$ & 0.0000 & 0.0007 & 0.0010 & 0.0007 & 0.0003 & 0.0007 & 0.0000 & 0.0007 & 0.0003 & 0.0007 & 0.0000 & 0.0007 \\
\hline $\mathrm{Sr}$ & 0.0049 & 0.0007 & 0.0023 & 0.0007 & 0.0064 & 0.0008 & 0.0023 & 0.0007 & 0.0101 & 0.0009 & 0.0008 & 0.0007 \\
\hline $\mathrm{Pb}$ & 0.0000 & 0.0008 & 0.0000 & 0.0008 & 0.0000 & 0.0008 & 0.0000 & 0.0008 & 0.0000 & 0.0008 & 0.0006 & 0.0008 \\
\hline & & & & & & & & & & & & \\
\hline Silicate Oxides & & & & & & & & & & & & \\
\hline $\mathrm{Na}_{2} \mathrm{O}$ & 0.2439 & & 0.1345 & & 0.0464 & & 0.0185 & & 0.0870 & & 0.0256 & \\
\hline $\mathrm{K}_{2} \mathrm{O}$ & 0.1272 & & 0.0393 & & 0.0453 & & 0.0177 & & 0.0495 & & 0.0235 & \\
\hline $\mathrm{CaO}$ & 1.0827 & & 0.0000 & & 1.9769 & & 0.2893 & & 2.1116 & & 0.2250 & \\
\hline $\mathrm{MgO}$ & 0.0000 & & 0.0000 & & 0.0109 & & 0.0000 & & 0.0000 & & 0.0000 & \\
\hline $\mathrm{Al}_{2} \mathrm{O}_{3}$ & 0.6009 & & 0.2277 & & 0.2300 & & 0.0421 & & 0.2662 & & 0.0954 & \\
\hline $\mathrm{SiO}_{2}$ & 2.1831 & & 0.8906 & & 1.1312 & & 0.3437 & & 1.6946 & & 0.3294 & \\
\hline Silicates & 4.2378 & & 1.2921 & & 3.4407 & & 0.7113 & & 4.2090 & & 0.6989 & \\
\hline Oxides & & & & & & & & & & & & \\
\hline Oxides & & & & & & & & & & & & \\
\hline $\mathrm{TiO}_{2}$ & 0.0420 & & 0.0170 & & 0.0127 & & 0.0040 & & 0.0224 & & 0.0048 & \\
\hline $\mathrm{V}_{2} \mathrm{O}_{5}$ & 0.0004 & & 0.0007 & & 0.0000 & & 0.0007 & & 0.0000 & & 0.0000 & \\
\hline $\mathrm{MnO}_{2}$ & 0.0119 & & 0.0028 & & 0.0011 & & 0.0000 & & 0.0035 & & 0.0028 & \\
\hline $\mathrm{Fe}_{2} \mathrm{O}_{3}$ & 0.3820 & & 0.1473 & & 0.1348 & & 0.0406 & & 0.2136 & & 0.0429 & \\
\hline Oxides & 0.4363 & & 0.1678 & & 0.1486 & & 0.0453 & & 0.2394 & & 0.0506 & \\
\hline & & & & & & & & & & & & \\
\hline lonic Compounds & & & & & & & & & & & & \\
\hline $\mathrm{NH}_{4} \mathrm{NO}_{3}$ & 0.7573 & & 0.3368 & & 0.0785 & & 0.0342 & & 0.0970 & & 0.0551 & \\
\hline$\left(\mathrm{NH}_{4}\right)_{2} \mathrm{SO}_{4}$ & 0.1103 & & 0.3142 & & 0.4494 & & 0.4167 & & 0.2646 & & 0.2936 & \\
\hline $\mathrm{CaSO}_{4} .2\left(\mathrm{H}_{2} \mathrm{O}\right)$ & 1.3354 & & 0.6352 & & 0.1499 & & 0.1021 & & 0.1840 & & 0.0571 & \\
\hline $\mathrm{NaCl}$ & 0.8506 & & 0.3922 & & 0.0000 & & 0.0000 & & 0.0097 & & 0.0000 & \\
\hline $\mathrm{Na}_{2} \mathrm{SO}_{4}$ & 0.0000 & & 0.0026 & & 0.0000 & & 0.0000 & & 0.0000 & & 0.0000 & \\
\hline $\mathrm{K}_{2} \mathrm{SO}_{4}$ & 0.0000 & & 0.0000 & & 0.0000 & & 0.0000 & & 0.0000 & & 0.0000 & \\
\hline Carhon & & & & & & & & & & & & \\
\hline & & & & & & & & & & & & \\
\hline EC & 0.1173 & & 0.0095 & & 0.0652 & & 0.0407 & & 0.1302 & & 0.0675 & \\
\hline $\mathrm{OC}$ & 2.3461 & & 0.7927 & & 0.9213 & & 0.7141 & & 1.5578 & & 1.2892 & \\
\hline Sum of & 10.1911 & & 3.9431 & & 5.2536 & & 2.0644 & & 6.6917 & & 25120 & \\
\hline Measured Mass & 18.2612 & 0.9276 & $\begin{array}{l}0.3701 \\
5.3705\end{array}$ & 0.3144 & $\frac{5.2530}{6.2396}$ & 0.3522 & $\begin{array}{l}2.0044 \\
2.5801\end{array}$ & 0.2083 & 8.6938 & 0.4644 & $\begin{array}{l}2.5120 \\
2.1223\end{array}$ & 0.1949 \\
\hline $\mathrm{PM}_{2.5} / \mathrm{PM}_{10}$ & & 0.2 & & & & 0.4 & & & & 0.2 & & \\
\hline
\end{tabular}


Table 11. Chemical and normative mineral compositions for fall 2009, winter 2009/10 and spring 2010 samples.

\begin{tabular}{|c|c|c|c|c|c|c|c|c|c|c|c|c|}
\hline \multirow{5}{*}{\begin{tabular}{|l|} 
SITE \\
TID \\
DATE \\
SIZE \\
\end{tabular}} & \multirow{2}{*}{\multicolumn{4}{|c|}{ Ash Meadows NWR }} & \multicolumn{4}{|c|}{ Ash Meadows NWR } & \multicolumn{4}{|c|}{ Ash Meadows NWR } \\
\hline & & & & & & YUCT & & & & YUCT & & \\
\hline & \multicolumn{4}{|c|}{$\begin{array}{l}\text { YUCT1152 } \\
11 / 09 / 09\end{array}$} & & $12 / 2$ & & & & $03 / 26$ & & \\
\hline & $\underline{P M}$ & & $\underline{\mathrm{PM}_{2}}$ & & PM & & $\underline{P M}$ & & PM & & PM & \\
\hline & Conc & Unc & Conc & Unc & Conc & Unc & Conc & Unc & Conc & Unc & Conc & Unc \\
\hline$\overline{\mathrm{Cl}^{-}}$ & 0.0000 & 0.0221 & 0.0000 & 0.0225 & 0.0000 & 0.0210 & 0.0000 & 0.0211 & 0.1610 & 0.0297 & 0.0000 & 0.0208 \\
\hline $\mathrm{NO}_{3}^{-}$ & 0.3488 & 0.0310 & 0.1074 & 0.0220 & 0.1532 & 0.0231 & 0.0753 & 0.0214 & 0.2305 & 0.0258 & 0.0982 & 0.0218 \\
\hline $\mathrm{SO}_{4}=$ & 0.6670 & 0.0396 & 0.5730 & 0.0357 & 0.2436 & 0.0242 & 0.1918 & 0.0230 & 2.0760 & 0.1070 & 0.3865 & 0.0285 \\
\hline $\mathrm{NH}_{4}^{+}$ & 0.2246 & 0.0242 & 0.2172 & 0.0240 & 0.0955 & 0.0215 & 0.0885 & 0.0214 & 0.0708 & 0.0212 & 0.1128 & 0.0217 \\
\hline $\mathrm{Na}^{+}$ & 0.0912 & 0.0055 & 0.0307 & 0.0022 & 0.0344 & 0.0024 & 0.0105 & 0.0014 & 1.2074 & 0.0712 & 0.0871 & 0.0053 \\
\hline $\mathrm{Mg}^{2+}$ & 0.1685 & 0.0085 & 0.0229 & 0.0014 & 0.0366 & 0.0020 & 0.0049 & 0.0009 & 0.0914 & 0.0047 & 0.0158 & 0.0012 \\
\hline $\mathrm{K}^{+}$ & 0.0807 & 0.0048 & 0.0303 & 0.0026 & 0.0240 & 0.0024 & 0.0117 & 0.0022 & 0.0673 & 0.0041 & 0.0215 & 0.0024 \\
\hline $\mathrm{Ca}^{2+}$ & 3.3290 & 0.1700 & 0.2885 & 0.0180 & 0.5531 & 0.0300 & 0.0974 & 0.0115 & 1.4146 & 0.0728 & 0.1614 & 0.0133 \\
\hline O1TC & 0.0000 & 0.0147 & 0.0709 & 0.0381 & 0.0000 & 0.0147 & 0.0326 & 0.0219 & 0.0043 & 0.0149 & 0.1483 & 0.0750 \\
\hline O2TC & 0.2144 & 0.0302 & 0.2291 & 0.0305 & 0.2502 & 0.0310 & 0.1768 & 0.0294 & 0.3791 & 0.0348 & 0.3978 & 0.0354 \\
\hline ОЗТC & 0.3016 & 0.0996 & 0.3143 & 0.1003 & 0.2752 & 0.0983 & 0.7432 & 0.1334 & 0.3994 & 0.1054 & 0.2699 & 0.0980 \\
\hline O4TC & 0.4384 & 0.1347 & 0.1033 & 0.0472 & 0.0937 & 0.0454 & 0.2156 & 0.0732 & 0.2380 & 0.0792 & 0.0724 & 0.0418 \\
\hline OPTTC & 0.3426 & 0.0814 & 0.0935 & 0.0335 & 0.0423 & 0.0278 & 0.0592 & 0.0293 & 0.1389 & 0.0408 & 0.0284 & 0.0269 \\
\hline OPTRC & 0.2295 & 0.0571 & 0.0490 & 0.0283 & 0.0877 & 0.0325 & 0.0000 & 0.0261 & 0.0858 & 0.0323 & 0.0222 & 0.0266 \\
\hline OC & 1.1839 & 0.1993 & 0.7666 & 0.1646 & 0.7068 & 0.1603 & 1.1682 & 0.1979 & 1.1066 & 0.1924 & 0.9105 & 0.1756 \\
\hline E1TC & 0.3426 & 0.0345 & 0.1153 & 0.0218 & 0.0423 & 0.0199 & 0.3108 & 0.0324 & 0.1512 & 0.0233 & 0.0284 & 0.0197 \\
\hline E2TC & 0.1586 & 0.0411 & 0.0271 & 0.0252 & 0.0453 & 0.0263 & 0.1319 & 0.0368 & 0.1273 & 0.0361 & 0.0205 & 0.0249 \\
\hline E3TC & 0.0000 & 0.0082 & 0.0000 & $\begin{array}{l}0.0082 \\
\end{array}$ & 0.0000 & 0.0082 & 0.0000 & 0.0082 & 0.0000 & 0.0082 & 0.0000 & 0.0082 \\
\hline ECTRC & 0.2718 & 0.0632 & 0.0935 & 0.0364 & 0.0000 & 0.0310 & 0.4427 & 0.0950 & 0.1928 & 0.0499 & 0.0267 & 0.0315 \\
\hline TC & 1.4557 & 0.2414 & 0.8601 & 0.1881 & 0.7068 & 0.1771 & 1.6109 & 0.2572 & 1.2995 & 0.2262 & 0.9372 & 0.1941 \\
\hline $\mathrm{Na}$ & 0.1061 & 0.0690 & 0.0000 & 0.0640 & 0.0000 & 0.0632 & 0.0930 & 0.0686 & 0.0000 & 0.0659 & 0.0369 & 0.0670 \\
\hline $\mathrm{Mg}$ & 0.0081 & 0.0213 & 0.0073 & 0.0213 & 0.0000 & 0.0211 & 0.0000 & 0.0212 & 0.0000 & 0.0208 & 0.0000 & 0.0211 \\
\hline Al & 0.0597 & 0.0054 & 0.2107 & 0.0117 & 0.0435 & 0.0049 & 0.0094 & 0.0043 & 0.2770 & 0.0149 & 0.1153 & 0.0075 \\
\hline $\mathrm{Si}$ & 0.2218 & 0.0116 & 0.8578 & 0.0433 & 0.2510 & 0.0130 & 0.0546 & 0.0040 & 1.0171 & 0.0513 & 0.4036 & 0.0206 \\
\hline S & 0.2092 & 0.0105 & 0.2058 & 0.0104 & 0.1035 & 0.0053 & 0.0729 & 0.0038 & 0.3777 & 0.0190 & 0.1562 & 0.0079 \\
\hline $\mathrm{Cl}$ & 0.0000 & 0.0007 & 0.0059 & 0.0008 & 0.0031 & 0.0007 & 0.0000 & 0.0007 & 0.0663 & 0.0034 & 0.0167 & 0.0011 \\
\hline $\mathrm{K}$ & 0.0497 & 0.0027 & 0.1348 & 0.0068 & 0.0446 & 0.0024 & 0.0129 & 0.0012 & 0.1604 & 0.0081 & 0.0590 & 0.0031 \\
\hline $\mathrm{Ca}$ & 0.2025 & 0.0107 & 0.6639 & 0.0335 & 0.3062 & 0.0158 & 0.0158 & 0.0032 & 0.6153 & 0.0311 & 0.1248 & 0.0071 \\
\hline $\mathrm{Ti}$ & 0.0045 & 0.0011 & 0.0199 & 0.0015 & 0.0054 & 0.0011 & 0.0007 & 0.0010 & 0.0216 & 0.0015 & 0.0068 & 0.0011 \\
\hline V & 0.0000 & 0.0007 & 0.0006 & 0.0007 & 0.0002 & 0.0007 & 0.0000 & 0.0007 & 0.0000 & 0.0007 & 0.0000 & 0.0007 \\
\hline $\mathrm{Mn}$ & 0.0000 & 0.0021 & 0.0032 & 0.0022 & 0.0020 & 0.0021 & 0.0002 & 0.0021 & 0.0058 & 0.0022 & 0.0011 & 0.0021 \\
\hline $\mathrm{Fe}$ & 0.0452 & 0.0024 & 0.2493 & 0.0125 & 0.0556 & 0.0029 & 0.0128 & 0.0010 & 0.2267 & 0.0114 & 0.0750 & 0.0038 \\
\hline $\mathrm{Cu}$ & 0.0010 & 0.0025 & 0.0029 & 0.0025 & 0.0021 & 0.0025 & 0.0005 & 0.0025 & 0.0021 & 0.0025 & 0.0007 & 0.0025 \\
\hline $\mathrm{Zn}$ & 0.0000 & 0.0007 & 0.0038 & 0.0008 & 0.0000 & 0.0007 & 0.0000 & 0.0007 & 0.0000 & 0.0007 & 0.0000 & 0.0007 \\
\hline $\mathrm{Br}$ & 0.0027 & 0.0007 & 0.0028 & 0.0007 & 0.0005 & 0.0007 & 0.0002 & 0.0007 & 0.0007 & 0.0007 & 0.0000 & 0.0007 \\
\hline $\mathrm{Rb}$ & 0.0000 & 0.0007 & 0.0004 & 0.0007 & 0.0000 & 0.0007 & 0.0003 & 0.0007 & 0.0003 & 0.0007 & 0.0000 & 0.0007 \\
\hline $\mathrm{Sr}$ & 0.0012 & 0.0007 & 0.0035 & 0.0007 & 0.0014 & 0.0007 & 0.0003 & 0.0007 & 0.0085 & 0.0008 & 0.0026 & 0.0007 \\
\hline $\mathrm{Pb}$ & 0.0000 & 0.0008 & 0.0000 & 0.0008 & 0.0000 & 0.0008 & 0.0000 & 0.0008 & 0.0000 & 0.0008 & 0.0000 & 0.0008 \\
\hline & & & & & & & & & & & & \\
\hline Silicate Oxides & & & & & & & & & & & & \\
\hline $\mathrm{Na}_{2} \mathrm{O}$ & 0.1229 & & 0.0414 & & 0.0464 & & 0.0142 & & 1.4868 & & 0.1174 & \\
\hline $\mathrm{K}_{2} \mathrm{O}$ & 0.0972 & & 0.0365 & & 0.0289 & & 0.0141 & & 0.0811 & & 0.0259 & \\
\hline $\mathrm{CaO}$ & 4.4599 & & 0.3581 & & 0.7108 & & 0.1278 & & 0.7731 & & 0.1311 & \\
\hline $\mathrm{MgO}$ & 0.0134 & & 0.0121 & & 0.0000 & & 0.0000 & & 0.0000 & & 0.0000 & \\
\hline $\mathrm{Al}_{2} \mathrm{O}_{3}$ & 0.1128 & & 0.3981 & & 0.0822 & & 0.0178 & & 0.5234 & & 0.2179 & \\
\hline $\mathrm{SiO}_{2}$ & 0.4744 & & 1.8347 & & 0.5368 & & 0.1168 & & 2.1754 & & 0.8632 & \\
\hline Silicates & 5.2807 & & 2.6810 & & 1.4052 & & 0.2906 & & 5.0399 & & 1.3555 & \\
\hline Orides & & & & & & & & & & & & \\
\hline$\frac{\text { Oxides }}{T S}$ & & & & & & & & & & & & \\
\hline $\mathrm{TiO}_{2}$ & 0.0075 & & 0.0332 & & 0.0090 & & 0.0012 & & 0.0360 & & 0.0113 & \\
\hline $\mathrm{V}_{2} \mathrm{O}_{5}$ & 0.0000 & & 0.0021 & & 0.0007 & & 0.0000 & & 0.0000 & & 0.0000 & \\
\hline $\mathrm{MnO}_{2}$ & 0.0000 & & 0.0051 & & 0.0032 & & 0.0003 & & 0.0092 & & 0.0017 & \\
\hline $\mathrm{Fe}_{2} \mathrm{O}_{3}$ & 0.0646 & & 0.3564 & & 0.0795 & & 0.0183 & & 0.3241 & & 0.1072 & \\
\hline Oxides & 0.0721 & & 0.3968 & & 0.0924 & & 0.0198 & & 0.3693 & & 0.1203 & \\
\hline & & & & & & & & & & & & \\
\hline lonic Com & & & & & & & & & & & & \\
\hline $\mathrm{NH}_{4} \mathrm{NO}_{3}$ & 0.4503 & & 0.1386 & & 0.1978 & & 0.0972 & & 0.2976 & & 0.1268 & \\
\hline$\left(\mathrm{NH}_{4}\right)_{2} \mathrm{SO}_{4}$ & 0.4509 & & 0.6810 & & 0.1865 & & 0.2439 & & 0.0137 & & 0.3085 & \\
\hline $\mathrm{CaSO}_{4} .2\left(\mathrm{H}_{2} \mathrm{O}\right)$ & 0.6079 & & 0.1397 & & 0.1936 & & 0.0260 & & 3.7024 & & 0.2908 & \\
\hline $\mathrm{NaCl}$ & 0.0000 & & 0.0000 & & 0.0000 & & 0.0000 & & 0.2654 & & 0.0000 & \\
\hline $\mathrm{Na}_{2} \mathrm{SO}_{4}$ & 0.0000 & & 0.0000 & & 0.0000 & & 0.0000 & & 0.0000 & & 0.0000 & \\
\hline $\mathrm{K}_{2} \mathrm{SO}_{4}$ & 0.0000 & & 0.0000 & & 0.0000 & & 0.0000 & & 0.0000 & & 0.0000 & \\
\hline & & & & & & & & & & & & \\
\hline Carbon & & & & & & & & & & & & \\
\hline$\overline{\mathrm{EC}}$ & 0.2718 & & 0.0935 & & 0.0000 & & 0.4427 & & 0.1928 & & 0.0267 & \\
\hline $\mathrm{OC}$ & 1.1839 & & 0.7666 & & 0.7068 & & 1.1682 & & 1.1066 & & 0.9105 & \\
\hline & & & & & & & & & & & & \\
\hline Sum of Species & 8.3176 & & 4.8972 & & 2.7822 & & 2.2884 & & 10.9876 & & 3.1390 & \\
\hline Measured Mass & 8.2848 & 0.4454 & 3.8270 & 16 & 3.0379 & 0.2232 & 1.1236 & 0.1729 & 11.4393 & 0.5949 & 3.0795 & 246 \\
\hline $\mathrm{PM}_{2.5} / \mathrm{PM}_{10}$ & & 0.4 & & & & 0.3 & & & & 0.2 & & \\
\hline
\end{tabular}


Table 12. Chemical and normative mineral compositions for spring 2010 samples

\begin{tabular}{|c|c|c|c|c|c|c|c|c|}
\hline \multirow{5}{*}{\begin{tabular}{|l|} 
SITE \\
TID \\
DATE \\
SIZE \\
\end{tabular}} & \multicolumn{4}{|c|}{ Ash Meadows NWR } & \multicolumn{4}{|c|}{ Ash Meadows NWR } \\
\hline & \multirow{2}{*}{\multicolumn{4}{|c|}{$\begin{array}{c}\text { YUCTT164 } \\
\text { 04/07/10 }\end{array}$}} & \multicolumn{4}{|c|}{ YUCTT168 } \\
\hline & & & & & & $05 / 2$ & & \\
\hline & \multicolumn{2}{|c|}{$\underline{\mathrm{PM}_{10}}$} & \multicolumn{2}{|c|}{$\underline{\mathrm{PM}_{2.5}}$} & \multicolumn{2}{|c|}{$\mathrm{PM}_{10}$} & \multicolumn{2}{|c|}{$\underline{\mathrm{PM}_{2.5}}$} \\
\hline & Conc & Unc & Conc & Unc & Conc & Unc & Conc & Unc \\
\hline $\mathrm{Cl}^{-}$ & 0.0515 & 0.0219 & 0.0000 & 0.0209 & -99 & -99 & 0.0000 & 0.0209 \\
\hline $\mathrm{NO}_{3}{ }^{-}$ & 0.1132 & 0.0221 & 0.0844 & 0.0215 & -99 & -99 & 0.3489 & 0.0310 \\
\hline $\mathrm{SO}_{4}=$ & 0.6996 & 0.0410 & 0.2993 & 0.0257 & -99 & -99 & 0.6074 & 0.0371 \\
\hline $\mathrm{NH}_{4}^{+}$ & 0.0965 & 0.0215 & 0.1072 & 0.0216 & -99 & -99 & 0.2290 & 0.0243 \\
\hline $\mathrm{Na}^{+}$ & 0.2687 & 0.0159 & 0.0391 & 0.0026 & -99 & -99 & 0.1173 & 0.0070 \\
\hline $\mathrm{Mg}^{2+}$ & 0.0514 & 0.0027 & 0.0114 & 0.0010 & -99 & -99 & 0.0228 & 0.0014 \\
\hline $\mathrm{K}^{+}$ & 0.0276 & 0.0025 & 0.0142 & 0.0022 & -99 & -99 & 0.0240 & 0.0024 \\
\hline $\mathrm{Ca}^{2+}$ & 0.6918 & 0.0368 & 0.1051 & 0.0117 & -99 & -99 & 0.1700 & 0.0135 \\
\hline O1TC & 0.0858 & 0.0450 & 0.0747 & 0.0398 & -99 & -99 & 0.1347 & 0.0683 \\
\hline O2TC & 0.2463 & 0.0309 & 0.3736 & 0.0346 & -99 & -99 & 0.4042 & 0.0356 \\
\hline OЗTC & 0.3326 & 0.1013 & 0.3409 & 0.1018 & -99 & -99 & 0.3923 & 0.1048 \\
\hline O4TC & 0.1967 & 0.0684 & 0.0949 & 0.0456 & -99 & -99 & 0.1609 & 0.0597 \\
\hline OPTTC & 0.1353 & 0.0401 & 0.0225 & 0.0266 & -99 & -99 & 0.1339 & 0.0399 \\
\hline OPTRC & 0.0000 & 0.0261 & 0.0348 & 0.0273 & -99 & -99 & 0.0666 & 0.0300 \\
\hline $\mathrm{OC}$ & 0.8614 & 0.1717 & 0.9189 & 0.1763 & -99 & -99 & 1.1587 & 0.1970 \\
\hline E1TC & 0.0878 & 0.0209 & 0.0044 & 0.0196 & -99 & -99 & 0.1324 & 0.0225 \\
\hline E2TC & 0.0641 & 0.0279 & 0.0305 & 0.0253 & -99 & -99 & 0.0468 & 0.0264 \\
\hline E3TC & 0.0000 & 0.0082 & 0.0000 & 0.0082 & -99 & -99 & 0.0000 & 0.0082 \\
\hline ECTRC & 0.1519 & 0.0437 & 0.0000 & 0.0310 & -99 & -99 & 0.1125 & 0.0385 \\
\hline TC & 1.0133 & 0.2003 & 0.9189 & 0.1927 & -99 & -99 & 1.2712 & 0.2234 \\
\hline $\mathrm{Na}$ & 0.0000 & 0.0628 & 0.0000 & 0.0647 & 0.0592 & 0.0676 & 0.1177 & 0.0693 \\
\hline $\mathrm{Mg}$ & 0.0000 & 0.0211 & 0.0000 & 0.0211 & 0.0221 & 0.0214 & 0.0000 & 0.0211 \\
\hline Al & 0.1535 & 0.0091 & 0.0534 & 0.0052 & 0.2912 & 0.0156 & 0.0551 & 0.0052 \\
\hline $\mathrm{Si}$ & 0.5932 & 0.0301 & 0.2517 & 0.0131 & 1.0461 & 0.0528 & 0.2107 & 0.0111 \\
\hline$S$ & 0.2016 & 0.0102 & 0.1200 & 0.0061 & 0.3219 & 0.0162 & 0.2599 & 0.0131 \\
\hline $\mathrm{Cl}$ & 0.0145 & 0.0010 & 0.0027 & 0.0007 & 0.0694 & 0.0036 & 0.0044 & 0.0007 \\
\hline K & 0.0836 & 0.0043 & 0.0345 & 0.0020 & 0.1769 & 0.0089 & 0.0410 & 0.0023 \\
\hline $\mathrm{Ca}$ & 0.5004 & 0.0254 & 0.0758 & 0.0050 & 0.9764 & 0.0491 & 0.1322 & 0.0074 \\
\hline $\mathrm{Ti}$ & 0.0125 & 0.0012 & 0.0075 & 0.0011 & 0.0264 & 0.0017 & 0.0037 & 0.0011 \\
\hline V & 0.0000 & 0.0007 & 0.0000 & 0.0007 & 0.0000 & 0.0007 & 0.0001 & 0.0007 \\
\hline $\mathrm{Mn}$ & 0.0049 & 0.0022 & 0.0004 & 0.0021 & 0.0053 & 0.0022 & 0.0000 & 0.0021 \\
\hline $\mathrm{Fe}$ & 0.1330 & 0.0067 & 0.0625 & 0.0032 & 0.2801 & 0.0141 & 0.0552 & 0.0029 \\
\hline $\mathrm{Cu}$ & 0.0009 & 0.0025 & 0.0017 & 0.0025 & 0.0017 & 0.0025 & 0.0000 & 0.0025 \\
\hline$Z n$ & 0.0000 & 0.0007 & 0.0000 & 0.0007 & 0.0033 & 0.0008 & 0.0036 & 0.0008 \\
\hline $\mathrm{Br}$ & 0.0000 & 0.0007 & 0.0013 & 0.0007 & 0.0026 & 0.0007 & 0.0020 & 0.0007 \\
\hline $\mathrm{Rb}$ & 0.0001 & 0.0007 & 0.0000 & 0.0007 & 0.0000 & 0.0007 & 0.0000 & 0.0007 \\
\hline $\mathrm{Sr}$ & 0.0026 & 0.0007 & 0.0000 & 0.0007 & 0.0105 & 0.0009 & 0.0007 & 0.0007 \\
\hline $\mathrm{Pb}$ & 0.0000 & 0.0008 & 0.0000 & 0.0008 & 0.0016 & 0.0008 & 0.0000 & 0.0008 \\
\hline Silicate Oxides & & & & & & & & \\
\hline $\mathrm{Na}_{2} \mathrm{O}$ & 0.3172 & & 0.0527 & & 0.0000 & & 0.1581 & \\
\hline $\mathrm{K}_{2} \mathrm{O}$ & 0.0333 & & 0.0171 & & 0.0000 & & 0.0289 & \\
\hline $\mathrm{CaO}$ & 0.6583 & & 0.1008 & & 0.0000 & & 0.0814 & \\
\hline $\mathrm{MgO}$ & 0.0000 & & 0.0000 & & 0.0366 & & 0.0000 & \\
\hline $\mathrm{Al}_{2} \mathrm{O}_{3}$ & 0.2900 & & 0.1009 & & 0.5502 & & 0.1041 & \\
\hline $\mathrm{SiO}_{2}$ & 1.2688 & & 0.5383 & & 2.2374 & & 0.4507 & \\
\hline Silicates & 2.5676 & & 0.8098 & & 2.8243 & & 0.8232 & \\
\hline Oxides & & & & & & & & \\
\hline$\overline{\mathrm{TiO}_{2}}$ & 0.0209 & & 0.0125 & & 0.0440 & & 0.0062 & \\
\hline $\mathrm{V}_{2} \mathrm{O}_{5}$ & 0.0000 & & 0.0000 & & 0.0000 & & 0.0004 & \\
\hline $\mathrm{MnO}_{2}$ & 0.0078 & & 0.0006 & & 0.0084 & & 0.0000 & \\
\hline $\mathrm{Fe}_{2} \mathrm{O}_{3}$ & 0.1902 & & 0.0894 & & 0.4005 & & 0.0789 & \\
\hline Oxides & 0.2188 & & 0.1025 & & 0.4529 & & 0.0854 & \\
\hline Ionic Compounds & & & & & & & & \\
\hline $\mathrm{NH}_{4} \mathrm{NO}_{3}$ & 0.1461 & & 0.1090 & & -99 & & 0.4504 & \\
\hline$\left(\mathrm{NH}_{4}\right)_{2} \mathrm{SO}_{4}$ & 0.2328 & & 0.3027 & & -99 & & 0.4669 & \\
\hline $\mathrm{CaSO}_{4} 2\left(\mathrm{H}_{2} \mathrm{O}\right)$ & 0.9505 & & 0.1421 & & -99 & & 0.4803 & \\
\hline $\mathrm{NaCl}$ & 0.0849 & & 0.0000 & & -99 & & 0.0000 & \\
\hline $\mathrm{Na}_{2} \mathrm{SO}_{4}$ & 0.0000 & & 0.0000 & & -99 & & 0.0000 & \\
\hline $\mathrm{K}_{2} \mathrm{SO}_{4}$ & 0.0000 & & 0.0000 & & -99 & & 0.0000 & \\
\hline & & & & & & & & \\
\hline Carbon & & & & & & & & \\
\hline $\mathrm{EC}$ & 0.1519 & & 0.0000 & & -99 & & 0.1125 & \\
\hline OC & 0.8614 & & 0.9189 & & -99 & & 1.1587 & \\
\hline Sum of Species & 52139 & & 2.3849 & & -99 & & 3.5774 & \\
\hline \begin{tabular}{|l|} 
Measured Mass \\
\end{tabular} & 6.5308 & 0.3652 & 2.2888 & 0.1996 & 13.8577 & 0.7119 & 3.8686 & 0.2532 \\
\hline $\mathrm{PM}_{2.5} / \mathrm{PM}_{10}$ & & 0.3 & & & & 0.2 & & \\
\hline
\end{tabular}




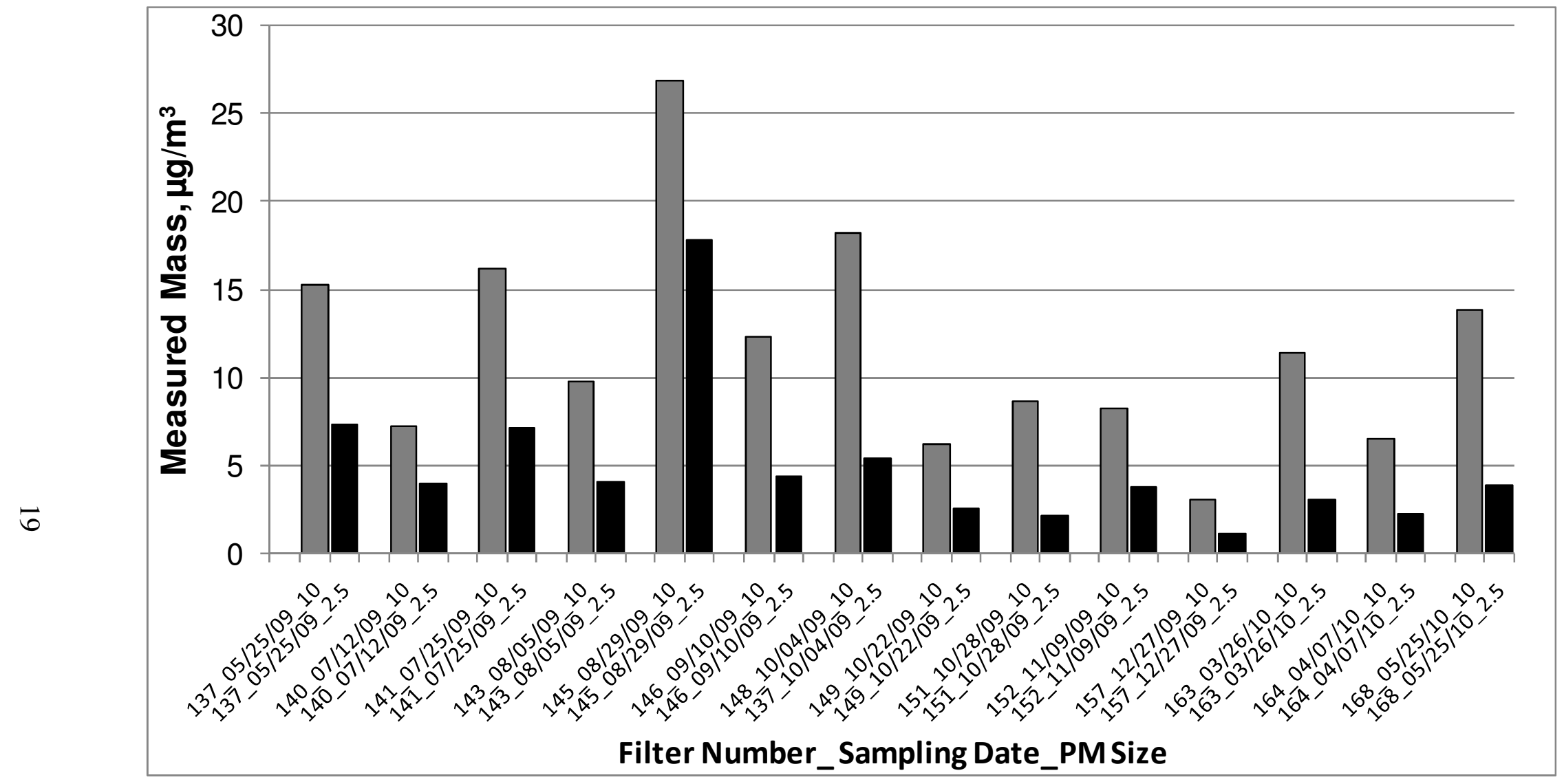

Figure 10. $\mathrm{PM}_{10}$ (gray) and $\mathrm{PM}_{2.5}$ (black) gravimetric data as measured on Teflon ${ }^{\circledR}$ filters, collected on a 1 -in-12 day schedule, for 14 individual sampling days. Sample labels include the sample numbers, sampling dates, and particulate size cut. 


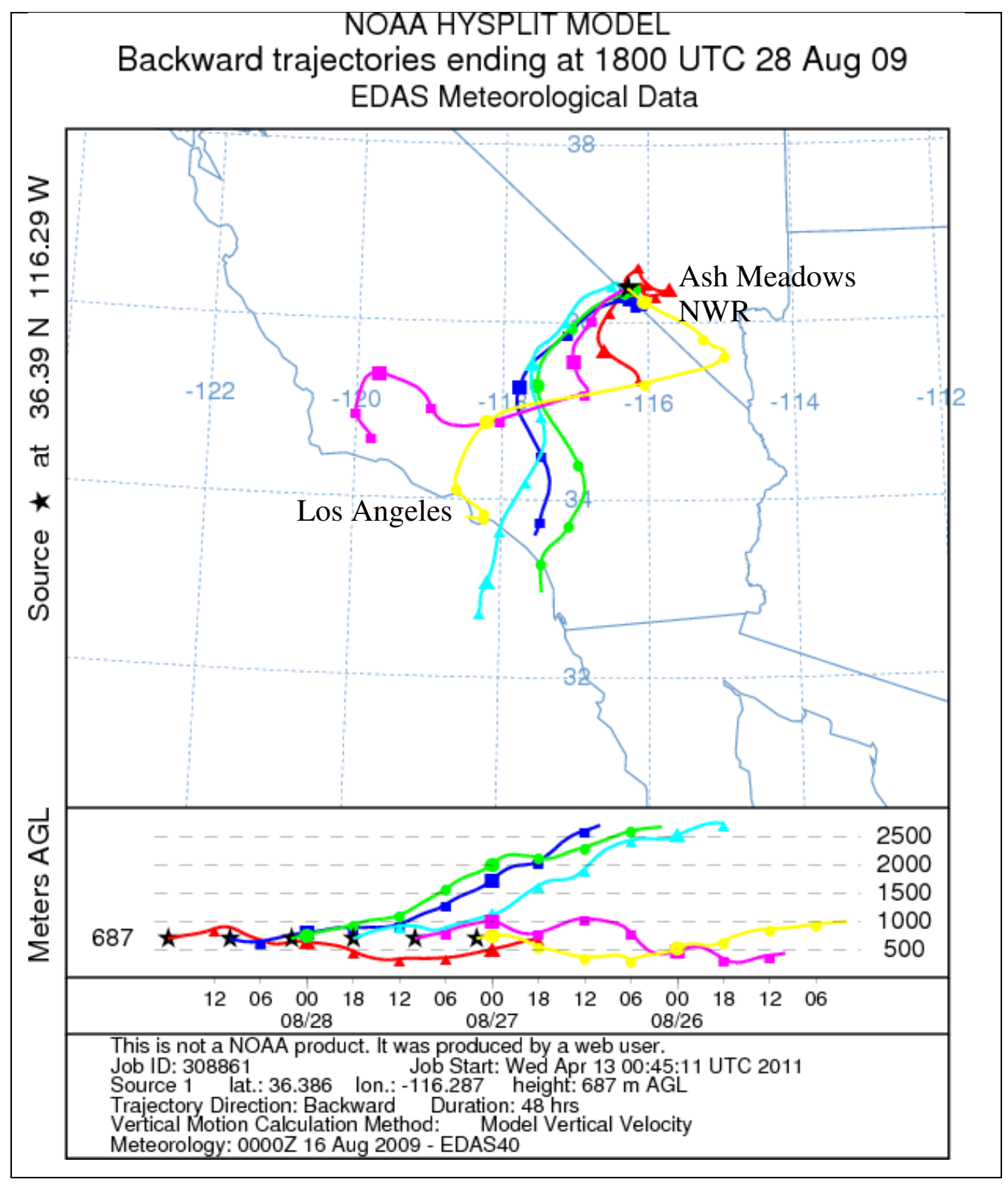

Figure 11. Hysplit backward trajectory model showing airflow from California towards Ash Meadows NWR for the August 27-29 period, coinciding to reported large wildfires in Los Angeles County during that period. 
Table 13 shows the average aerosol concentrations at Ash Meadows NWR being low in $\mathrm{PM}_{10}$, but variable by season. It was lowest in winter $\left(3 \mu \mathrm{g} / \mathrm{m}^{3}\right)$ and highest $\left(15 \mu \mathrm{g} / \mathrm{m}^{3}\right)$ in spring and summer. Similarly for $\mathrm{PM}_{2.5}$, the lowest concentrations were found in winter $\left(1 \mu \mathrm{g} / \mathrm{m}^{3}\right)$ with higher ( 4 to $9 \mu \mathrm{g} / \mathrm{m}^{3}$ ) values for the other three seasons. The PM levels are on average similar to those from the eight sites in southwestern Nevada and to the four IMPROVE sites. The measurements taken in the course of this Ash Meadows NWR campaign are substantially lower than those from the urban CSN sites. Both the $\mathrm{PM}_{10}$ and $\mathrm{PM}_{2.5}$ levels are substantially lower than the $24 \mathrm{hr}$ and the annual US EPA National Ambient Air Quality Standards (NAAQS).

Table 13. Comparison of seasonal average gravimetric results from this study (Ash Meadows NWR, 05/25/09 to 05/25/10) with those from the preceding study at eight sites in rural southwestern Nevada, urban southwestern U.S.A. (CSN) and rural southwestern U.S.A. (IMPROVE) sites.

\begin{tabular}{|c|c|c|c|c|c|c|}
\hline Region & Date & Date & Avg. & Avg. & $\mathrm{PM}_{2.5} / \mathrm{PM}_{10}$ & Days \\
\hline & Start & End & $\mathbf{P M}_{10}$ & $\mathbf{P M}_{2.5}$ & & of $24 \mathrm{hr}$ \\
\hline & & & $\mu g / m^{3}$ & $\mu g / m^{3}$ & & Data \\
\hline 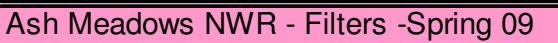 & $005 / 25 / 09$ & $005 / 25 / 09$ & 15 & $\overline{7}$ & 0.48 & $\overline{11}$ \\
\hline Ash Meadows NWR - Filters-Summer & 07/12/09 & 08/29/09 & 15 & 9 & 0.58 & 4 \\
\hline Ash Meadows NWR - Filters - Fall & 09/10/09 & $11 / 09 / 09$ & 11 & 4 & 0.34 & 5 \\
\hline Ash Meadows NWR - Filters Winter & $12 / 27 / 09$ & $12 / 27 / 09$ & 3 & 1 & 0.49 & 1 \\
\hline Ash Meadows NWR - Filters - Spring 10 & $03 / 26 / 10$ & 05/25/10 & 11 & 4 & 0.42 & 3 \\
\hline Ash Meadows NWR - Filters - Average & $05 / 25 / 09$ & $05 / 25 / 10$ & 11 & 5 & 0.47 & 14 \\
\hline S-W Nedvada - Filters - 8 sites & $03 / 24 / 06$ & $12 / 19 / 07$ & 10 & 4 & 0.47 & 86 \\
\hline S-W USA - 5 Urban CSN sites & 2006 & 2007 & 38 & 11 & 0.29 & $>500$ \\
\hline S-W USA - 4 Rural IMPROVE sites & $03 / 24 / 06$ & $12 / 19 / 07$ & 13 & 5 & 0.39 & $>500$ \\
\hline US EPA NAAQS $24 \mathrm{hr}$ & & & 150 & 35 & & \\
\hline US EPA NAAQS annual & & & & 15 & & \\
\hline
\end{tabular}

There are differences between the average $\mathrm{PM}_{10}$ gravimetric values measured on the Teflon ${ }^{\circledR}$ filters $\left(11 \mu \mathrm{g} / \mathrm{m}^{3}\right.$, Table 13$)$, compared to the TEOM ${ }^{\circledR}$ monitor $\left(9 \mu \mathrm{g} / \mathrm{m}^{3}\right.$, Table 3$)$. For $\mathrm{PM}_{2.5}$, the differences between Teflon ${ }^{\circledR}$ filters $\left(5 \mu \mathrm{g} / \mathrm{m}^{3}\right)$, and the $\mathrm{TEOM}^{\circledR}$ monitor $\left(4 \mu \mathrm{g} / \mathrm{m}^{3}\right)$ are less. These discrepancies between the TEOM ${ }^{\circledR}$ and Teflon ${ }^{\circledR}$ filter results are ascribed to differences in the instrumentation and sampling techniques - the TEOM ${ }^{\circledR}$ sampling chamber and filter stub are heated to $50{ }^{\circ} \mathrm{C}$, resulting in the evaporation of semi-volatiles such as water and organic compounds from the sampled particulate matter on the filter, with a resultant loss in mass.

\section{Filter Chemistry}

In the course of this study, Teflon ${ }^{\circledR}$ filters, including field and laboratory blanks were analyzed by Energy Dispersive X-ray Fluorescence Spectrometry (EDXRF) for 40 chemical elements including total sodium $(\mathrm{Na})$, magnesium $(\mathrm{Mg})$, aluminum $(\mathrm{Al})$, silicon $(\mathrm{Si})$, sulfur $(\mathrm{S})$, 
chlorine $(\mathrm{Cl})$, potassium $(\mathrm{K})$, calcium $(\mathrm{Ca})$, titanium $(\mathrm{Ti})$, vanadium $(\mathrm{V})$, manganese $(\mathrm{Mn})$, iron $(\mathrm{Fe})$, copper $(\mathrm{Cu})$, zinc $(\mathrm{Zn})$, bromine $(\mathrm{Br})$, rubidium $(\mathrm{Rb})$, strontium $(\mathrm{Sr})$, and lead $(\mathrm{Pb})$.

Water extractions were performed on one half of each of the quartz fiber filters, including field and laboratory blanks. Aliquots of the extractions were analyzed by Ion Chromatography (IC) for water soluble anions, sulfate $\left(\mathrm{SO}_{4}{ }^{2-}\right)$, nitrate $\left(\mathrm{NO}_{3}{ }^{-}\right)$, and chloride $\left(\mathrm{Cl}^{-}\right)$. An aliquot thereof was analyzed for the ammonium $\left(\mathrm{NH}_{4}{ }^{+}\right)$cations by Automated Colorimetry (AC). Further aliquots of the extractions were analyzed by Atomic Absorption (AA) for water soluble cations including sodium $\left(\mathrm{Na}^{+}\right)$, potassium $\left(\mathrm{K}^{+}\right)$, calcium $\left(\mathrm{Ca}^{2+}\right)$, and magnesium $\left(\mathrm{Mg}^{2+}\right)$.

Punches from the remaining half of each of the quartz fiber filters were analyzed by Thermal Optical Reflectance (TOR) for four fractions of organic carbon (OC) and three fractions of elemental carbon (EC), by the IMPROVE method (http://vista.cira.colostate.edu/IMPROVE /Overview/Overview.htm).

Tables 8 to 12 list the analytical results of 14 individual days of $24 \mathrm{hr}$ filter sampling at Ash Meadows NWR, for both $\mathrm{PM}_{10}$ and $\mathrm{PM}_{2.5}$, for each season. The chemical results were calculated for oxides and ionic compounds, and grouped into four categories: silicate oxides $\left(\mathrm{Na}_{2} \mathrm{O}, \mathrm{K}_{2} \mathrm{O}, \mathrm{CaO}, \mathrm{MgO}, \mathrm{Al}_{2} \mathrm{O}_{3}, \mathrm{SiO}_{2}\right)$, minor oxides $\left(\mathrm{TiO}_{2}, \mathrm{~V}_{2} \mathrm{O}_{5}, \mathrm{MnO}_{2}, \mathrm{Fe}_{2} \mathrm{O}_{3}\right)$, ionic compounds $\left(\mathrm{NH}_{4} \mathrm{NO}_{3},\left(\mathrm{NH}_{4}\right)_{2} \mathrm{SO}_{4}, \mathrm{CaSO}_{4} \cdot 2\left(\mathrm{H}_{2} \mathrm{O}\right), \mathrm{NaCl}, \mathrm{Na}_{2} \mathrm{SO}_{4}, \mathrm{~K}_{2} \mathrm{SO}_{4}\right)$, elemental carbon (EC), and organic carbon (OC).

The silicates (Figure 12) are a major component in all samples, varying from 23-66\% of the sample mass for $\mathrm{PM}_{10}$ and $10-55 \%$ of the sample mass for $\mathrm{PM}_{2.5}$, with larger fractions in the three samples collected in October and November 2009. This can be ascribed to airborne mineral dust prevailing during the late fall of 2009. Small amounts of gypsum were found in all samples, together with trace to minor amounts of evaporites such as halite and water soluble sulfates.

Secondary ammonium sulfate and secondary ammonium nitrate, both largely from combustion processes, occur mostly in the $\mathrm{PM}_{2.5}$ fractions, with the highest concentrations for both these secondary compounds in samples collected during the period May - October, 2009. High concentrations of both elemental carbon $\left(1.2 \mu \mathrm{g} / \mathrm{m}^{3}\right)$ and organic carbon $\left(8.5 \mu \mathrm{g} / \mathrm{m}^{3}\right)$ in $\mathrm{PM}_{10}$ with similar amounts in $\mathrm{PM}_{2.5}$ were analyzed in the $08 / 29 / 09$ sample, providing additional evidence of smoke from wildfires in Los Angeles County, California. 


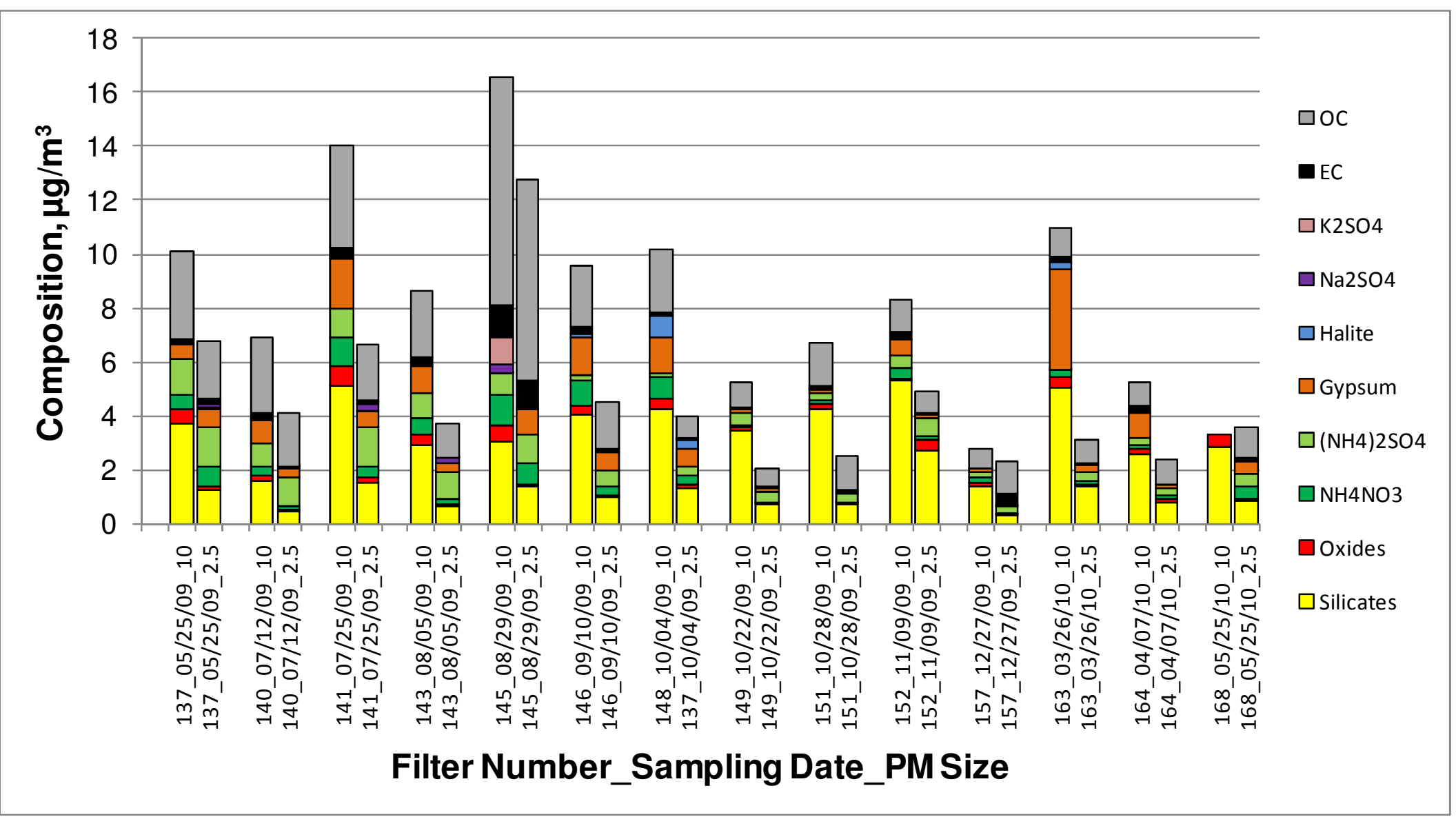

Figure 12. Chemical measurements combined as silicate oxides, minor metal oxides, ammonium sulfate, ammonium nitrate, gypsum, halite, sodium sulfate, potassium sulfate, elemental carbon (EC) and organic carbon (OC), for $\mathrm{PM}_{10}$ and associated $\mathrm{PM}_{2.5}$ size fractions. Sample labels include the sample numbers, sampling dates, and particulate size cut. 


\section{CONCLUSIONS}

- The instrumented trailer provided a versatile mobile platform for housing and transporting an array of air quality and meteorological instrumentation. Besides the two continuous particulate matter (PM) monitors $\left(\mathrm{TEOM}^{\circledR} \mathrm{s}\right)$, all instrumentation in the trailer could also be powered from photovoltaic charged $12 \mathrm{~V} \mathrm{DC}$ batteries.

- Measurements taken at Ash Meadows NWR over a period of 12 months in 2009 and 2010 provided baseline air quality and meteorological information for rural southwestern Nevada, specifically Nye County and the Amargosa Valley.

- There are inconsistent differences between the average $\mathrm{PM}_{10}$ gravimetric results from the Teflon ${ }^{\circledR}$ filters $\left(12 \mu \mathrm{g} / \mathrm{m}^{3}\right)$, and the TEOM ${ }^{\circledR}$ monitors $\left(9 \mu \mathrm{g} / \mathrm{m}^{3}\right)$. There is less of a difference for $\mathrm{PM}_{2.5}$, being on average $5 \mu \mathrm{g} / \mathrm{m}^{3}$ for the Teflon ${ }^{\circledR}$ filters and $4 \mu \mathrm{g} / \mathrm{m}^{3}$ for the TEOM ${ }^{\circledR}$ monitor. The corresponding $\mathrm{PM}_{2.5} / \mathrm{PM}_{10}$ ratios vary between the 0.42 for the Teflon $^{\circledR}$ filters and 0.36 for the corresponding TEOM ${ }^{\circledR}$ measurements. These discrepancies are attributed to the loss of semi-volatile organic compounds and water from the heated $\mathrm{TEOM}^{\circledR}$ 's sampling stub.

- All mass measurements fall well below the NAAQS for $\mathrm{PM}_{10}\left(150 \mu \mathrm{g} / \mathrm{m}^{3}\right.$ for $24 \mathrm{hr}$ averaging interval) and $\mathrm{PM}_{2.5}\left(35 \mu \mathrm{g} / \mathrm{m}^{3}\right.$ for $24 \mathrm{hr}$ averaging interval, $15 \mu \mathrm{g} / \mathrm{m}^{3}$ for annual averaging interval). The averaged levels measured on Teflon ${ }^{\circledR}$ at Ash Meadows NWR for both $\mathrm{PM}_{10}\left(12 \mu \mathrm{g} / \mathrm{m}^{3}\right)$ and $\mathrm{PM}_{2.5}\left(5 \mu \mathrm{g} / \mathrm{m}^{3}\right)$, are similar to those previously measured at four rural IMPROVE sites.

- The mineral dust (soil) components, including the silicate oxides, minor oxides and gypsum, together make up the largest percentage of the aerosol, being on average $58 \%$ of $\mathrm{PM}_{10}$ by mass and $37 \%$ of $\mathrm{PM}_{2.5}$ by mass, the difference being ascribed to the coarser nature of the soil-forming minerals. The highest concentrations of mineral dust components were measured during drier fall months of October and November, 2009.

- Although halite $(\mathrm{NaCl})$ was found in all samples, the percentages are always very low, except for the filter samples collected on 10/04/2009. The anomalously high values of $8 \%$ halite for $\mathrm{PM}_{10}$ and $10 \%$ for $\mathrm{PM}_{2.5}$ point to an evaporite (salt) source such as an alkaline playa dust. Playas are common in the region around Ash Meadows NWR.

- Although secondary ammonium sulfate concentrations vary with season, they are, within margins of uncertainty, similar for $\mathrm{PM}_{10}$ and $\mathrm{PM}_{2.5}$, implying that this species occurs in the finer fraction. Average values for $\mathrm{PM}_{10}$ were $0.5 \mu \mathrm{g} / \mathrm{m}^{3}$, and $0.7 \mu \mathrm{g} / \mathrm{m}^{3}$ for $\mathrm{PM}_{2.5}$, with the highest concentrations in spring 2009 and the lowest in following late fall and winter. Secondary ammonium sulfate is from $\mathrm{SO}_{2}$ emitted by distant power plants and other combustion sources, and transported into the Amargosa Valley. 
- Ammonium nitrate concentrations fluctuate by the month with both the lowest $(0.08$ $\mu \mathrm{g} / \mathrm{m}^{3}$ for $\mathrm{PM}_{10}$ and $0.03 \mu \mathrm{g} / \mathrm{m}^{3}$ for $\left.\mathrm{PM}_{2.5}\right)$ and highest $\left(1.11 \mu \mathrm{g} / \mathrm{m}^{3}\right.$ for $\mathrm{PM}_{10}$ and 0.77 $\mu \mathrm{g} / \mathrm{m}^{3}$ for $\mathrm{PM}_{2.5}$ ) measured values being in fall of 2009. Secondary nitrates form from nitrogen oxides emitted from vehicle emissions, possibly transported to Amargosa Valley from the Las Vegas - Los Angeles corridor.

- The elemental carbon (EC) is generally very low (on average $<0.2 \mu \mathrm{g} / \mathrm{m}^{3}$ ) except for the samples collected on 8/29/2009 when high values for EC of $1.20 \mu \mathrm{g} / \mathrm{m}^{3}$ for $\mathrm{PM}_{10}$ and $1.04 \mu \mathrm{g} / \mathrm{m}^{3}$ for $\mathrm{PM}_{2.5}$ were measured. Similarly, OC was low (on average $<1.8 \mu \mathrm{g} / \mathrm{m}^{3}$ ) except for the sample of $8 / 29 / 2009$ when high values for OC of $8.5 \mu \mathrm{g} / \mathrm{m}^{3}$ for $\mathrm{PM}_{10}$ and $7.5 \mu \mathrm{g} / \mathrm{m}^{3}$ for $\mathrm{PM}_{2.5}$ were measured. These elevated carbon levels are attributed to woodsmoke from wildfires in Los Angeles County, California.

\section{ACKNOWLEDGEMENTS}

- We thank the following for providing assistance and support with the deployment of the trailer:

U.S. Fish \& Wildlife Service and their personnel at Ash Meadows NWR

- Funding for this study was provided by the U.S. Department of Energy, National Nuclear Security Administration, Nevada Site Office through Contract No. DE-AC5206NA25383 to DRI.

\section{REFERENCES}

Belcher, W.R. (ed). 2004. Death Valley regional ground-water flow system, Nevada and California-hydrogeologic framework and transient groundwater flow model. United States Geological Survey Scientific Investigation Report 04-5205.

Chow, J. C., and Watson, J. G., 1999, Ion Chromatography in elemental analysis of airborne particles: Elemental Analysis of Airborne Particles, v. vol. 1: Amsterdam, Gordon and Breach Science, 97-137 p.

Chow, J. C., Watson, J. G., Pritchett, L. C., Pierson, W. R., and Purcell, R. G., 1993, The DRI Thermal/Optical Reflectance carbon analysis system: Description, evaluation and applications in U.S. air quality studies: Atmospheric Envirionment, v. 27A, p. 1185-1201.

Engelbrecht , J. P., Kavouras, I., Campbell, D., Campbell, S., Kohl, S. D., and Shafer, D. S., 2007a, Air Quality Scoping Study for Ash Meadows National Wildlife Refuge, Nevada, Letter Report DOE/NV/26383-LTR2007-01, Desert Research Institute, p. 1-25.

—, 2007b, Air Quality Scoping Study for Beatty, Nevada, Letter Report DOE/NV/26383LTR2007-02, Desert Research Institute, p. 1-25.

—, 2007c, Air Quality Scoping Study for Rachel, Nevada, Letter Report DOE/NV/26383LTR2007-03, Desert Research Institute, p. 1-26.

—, 2007d, Air Quality Scoping Study for Sarcobatus Flat, Nevada, Letter Report DOE/NV/26383-LTR2007-04, Desert Research Institute, p. 1-25. 
—, 2008a, Air Quality Scoping Study for Caliente, Lincoln County, Nevada, Letter Report DOE/NV/26383-LTR2008-01, Desert Research Institute, p. 1-25.

—, 2008b, Air Quality Scoping Study for Crater Flat, Nye County, Nevada, Letter Report DOE/NV/26383-LTR2008-03, Desert Research Institute, p. 1-22.

—, 2008c, Air Quality Scoping Study for Pahranagat National Wildlife Refuge, Lincoln County, Nevada, Letter Report DOE/NV/26383-LTR2008-02, Desert Research Institute, p. 1-25.

—, 2008d, Air Quality Scoping Study for Tonopah Airport, Nye County, Nevada, Letter Report DOE/NV/26383-LTR2008-04, Desert Research Institute, p. 1-25.

Engelbrecht , J. P., Kavouras, I., Shafer, D. S., Campbell, D., Campbell, S., McCurdy, G., Kohl, S. D., Nikolich, G., Sheetz, L., and Gertler, A. W., 2011, Air quality measurements at eight sites in rural southwestern Nevada, U.S.A.: Journal of the Air \& Waste Management Association, v. (in preparation)

Watson, J. G., Chow, J. C., and Frazier, C. A., 1999, X-ray fluorescence analysis of ambient air samples, in Cohen, B. S., and Herring, S. V., eds., Air Sampling Instruments for Evaluation of Atmospheric Contaminants, Cincinnati, OH, p. 51-57. 